\title{
Implementation and Scalability Analysis of Balancing Domain Decomposition Methods
}

\author{
Santiago Badia · Alberto F. Martín · Javier Principe
}

Received: 10 March 2013 / Accepted: 10 March 2013 / Published online: 2 July 2013

(C) CIMNE, Barcelona, Spain 2013

\begin{abstract}
In this paper we present a detailed description of a high-performance distributed-memory implementation of balancing domain decomposition preconditioning techniques. This coverage provides a pool of implementation hints and considerations that can be very useful for scientists that are willing to tackle large-scale distributed-memory machines using these methods. On the other hand, the paper includes a comprehensive performance and scalability study of the resulting codes when they are applied for the solution of the Poisson problem on a large-scale multicorebased distributed-memory machine with up to 4096 cores. Well-known theoretical results guarantee the optimality (algorithmic scalability) of these preconditioning techniques for weak scaling scenarios, as they are able to keep the condition number of the preconditioned operator bounded by a constant with fixed load per core and increasing number of cores. The experimental study presented in the paper complements this mathematical analysis and answers how far can these methods go in the number of cores and the scale of the problem to still be within reasonable ranges of efficiency on current distributed-memory machines. Besides, for those scenarios where poor scalability is expected, the
\end{abstract}

S. Badia $(\bowtie) \cdot$ A.F. Martín · J. Principe

Centre Internacional de Mètodes Numèrics a l'Enginyeria

(CIMNE), Parc Mediterrani de la Tecnologia, UPC, Esteve

Terradas 5, 08860 Castelldefels, Spain

e-mail: sbadia@cimne.upc.edu

A.F. Martín

e-mail: amartin@cimne.upc.edu

J. Principe

e-mail: principe@cimne.upc.edu

S. Badia · A.F. Martín · J. Principe

Universitat Politècnica de Catalunya, Jordi Girona 1-3, Edifici C1, 08034 Barcelona, Spain study precisely identifies, quantifies and justifies which are the main sources of inefficiency.

\section{Introduction}

Scientific phenomena governed by partial differential equations (PDEs) can range from solid mechanics to fluid mechanics and electrodynamics, including any of the possible couplings. The solution of these equations can be approximated with the aid of computers by a discretization (and possibly linearization) and the subsequent numerical solution of the resulting sparse set of linear equations. This work is concerned with the fast solution of the Poisson problem discretized by the finite element (FE) method. Although the Poisson problem is the simplest model problem for, e.g., fluid flow simulation, it is still very useful as a building block for the "physics-based" preconditioning of very complex scientific applications governed by coupled systems of PDEs [1].

The ever increasing demand of reality in the simulation of the complex scientific and engineering three-dimensional (3D) problems faced nowadays ends up with the solution of very large and sparse linear systems with several hundreds and even thousands of millions of equations/unknowns. The solution of these systems in a moderate time requires the vast amount of computational resources provided by current multicore-based distributed-memory machines. It is therefore essential to design parallel algorithms able to take profit of their underlying architecture.

Non-overlapping domain decomposition (DD) methods (also referred as iterative sub-structuring or Schur complement methods) provide a natural framework for the development of fast parallel solvers tailored for distributed-memory machines, as they have by construction the desirable design 
principle of maximizing local computations while minimizing interprocessor communication. One-level DD preconditioners, such as the Neumann-Neumann preconditioner, are highly parallel as they only require the solution of local problems and communication among neighboring subdomains, but unfortunately they do not posses optimality properties. Consequently, e.g., in a weak scaling scenario, where the number of processors is increased while keeping the load per processor constant, more a more computational resources are wasted because extra iterations are required to converge. Two-level DD preconditioners combine local and global terms acting in an additive or in a multiplicative fashion in order to achieve quasi-optimal condition number bounds (in the sense discussed in the next paragraph). The global term couples all the subdomains and involves the solution of a "small" (relative to the original linear system) coarse-grid problem. Besides, its size has typically only a linear dependence with the number of processors. However, only a small amount of parallelism can be exploited for the solution of this coarse-grid problem, which results in increasing parallel overheads (i.e., loss of efficiency) with the number of processors.

In this work we focus on two-level DD methods of balancing type, namely the Balancing Neumann-Neumann [28]
(BNN) and Balancing DD by Constraints [10] (BDDC)
methods; other two-level DD preconditioners ard found
in the FETI family [11, 12], although they are not ex-
plored here. These methods are quasi-optimal (algorithmi-
caly scalable) with a poly-logarithmic expression of the condition number of the preconditioned system $\kappa=1+$ $\log ^{2}\left(\frac{H}{h}\right)$, where $h$ and $H$ are, respectively, the mesh and

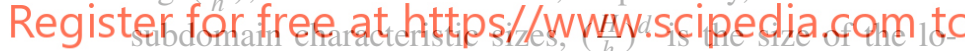
cal problems and $d$ is the space dimension. Consequently, in weak scaling scenarios (i.e., $\frac{H}{h}$ fixed), the number of iterations of the preconditioned conjugate gradient (PCG) solver is (asymptotically) independent of the number of processors.

Even though the mathematical theory of these methods is well established, there is a surprising lack of scientific works on the design/implementation issues to be considered for the efficient exploitation of distributed-memory machines. And even more surprising is the lack of comprehensive performance and scalability analysis on large-scale distributed-memory machines. This situation is in contrast to multigrid methods, see e.g. [3, 27]. We believe that the ability of balancing DD (BDD) methods to exploit large-scale distributed-memory machines is the most cited feature but their least examined one. To the best of our knowledge only few studies focus on these aspects for this particular family of algorithms [7, 19, 20,38], none of which with the degree of detail and up to the scale that are considered here. Given this lack, the contribution of this paper to the state-of-the-art is twofold. On the one hand, we present a comprehensive coverage of design/implementation issues provided by the experience we have acquired by implementing them from scratch in our FE/numerical linear algebra library which results in part from preliminary scalability studies. This coverage is intended to provide scientists with some hints and issues that have to take into account if they want to tackle large-scale problems efficiently on distributed-memory machines. On the other hand, we present a comprehensive weak scalability study of this implementation on a distributedmemory machine with up to 4096 cores. The main objective of this study is to identify and quantify sources of overhead in our current implementation (mainly the impact of the coarse-grid solver) and determine to what degree they are weakly scalable, i.e., how far can these methods go in the number of cores and the scale of the problem to still be within reasonable ranges of efficiency.

The article is organized as follows. In Sect. 2 we present the basic ideas underlying BDD preconditioners. For theoretical aspects of the algorithms we refer the reader to the vast literature devoted to DD methods; see, e.g, [42] and references therein. Our high-performance distributed-memory implementation of these methods is described in Sect. 3, and Sect. 4 presents the aforementioned scalability study. Finally, some concluding remarks are enumerated in Sect. 5.

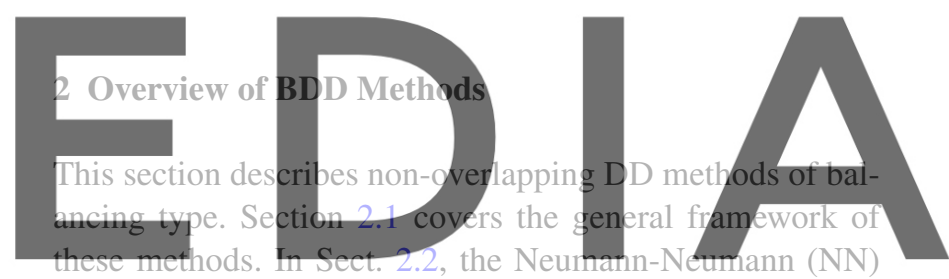

preconditioner $[9,18]$ is presented. Although this precon-

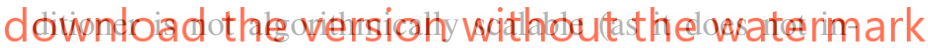
clude a coarse-grid correction), it is the basis for the more sophisticated BNN preconditioner [28], which is covered in Sect. 2.3. Finally, the widely used BDDC [10] preconditioning technique is described in Sect. 2.4.

\subsection{General Framework}

As model problem, let us consider the Poisson problem on a domain $\Omega \subset \mathbb{R}^{d}$, with homogeneous Dirichlet boundary conditions on $\partial \Omega$, where $d=2,3$ is the number of space dimensions. We also consider a uniform FE partition (mesh) $\mathcal{T}=\left\{K_{i}: i=1, \ldots, n_{\text {elm }}\right\}$ of $\Omega$ with characteristic size $h$. We are interested in solving the set of linear equations

$A x=b$,

which arises from the Galerkin FE discretization of the continuous problem corresponding to $\mathcal{T}$.

Further, we consider a uniform non-overlapping partition of $\Omega$ into subdomains $\left\{\Omega_{i}: i=1, \ldots, n_{\mathrm{sbd}}\right\}$ with characteristic size $H$ and a partition of the global mesh $\mathcal{T}$ into local meshes $\left\{\mathcal{T}_{i}: i=1, \ldots, n_{\text {sbd }}\right\}$ such that $\mathcal{T}_{i}$ is a conforming 
mesh of $\Omega_{i}$. The interface of $\Omega_{i}$ is defined as $\Gamma_{i}=\partial \Omega_{i} \backslash \partial \Omega$ and the whole interface (skeleton) of the domain decomposition is $\Gamma=\bigcup_{i=1}^{n_{\mathrm{sbd}}} \Gamma_{i}$. The set of nodes of $\mathcal{T}$ that belong to $\Gamma$ (resp. $\Gamma_{i}$ ) is denoted by $\Gamma_{h}$ (resp. $\Gamma_{h}^{i}$ ). This partition of the domain into non-overlapping subdomains induces the following block reordered structure of(1):

$\left[\begin{array}{ll}A_{I I} & A_{I \Gamma} \\ A_{\Gamma I} & A_{\Gamma \Gamma}\end{array}\right]\left[\begin{array}{c}x_{I} \\ x_{\Gamma}\end{array}\right]=\left[\begin{array}{l}b_{I} \\ b_{\Gamma}\end{array}\right]$,

where $x_{\Gamma}$ contains the unknowns corresponding to the nodes in $\Gamma_{h}$ and $x_{I}$ the remaining ones, associated with subdomain interiors. Besides, $A_{I I}$ presents a block diagonal structure (and therefore very amenable to parallelization), i.e.,

$A_{I I}=\operatorname{diag}\left(A_{I I}^{1}, A_{I I}^{2}, \ldots, A_{I I}^{n_{\text {sbd }}}\right)$,

where $A_{I I}^{i}$ is the local matrix which represents the coupling of internal unknowns at subdomain $i$. Eliminating $x_{I}$ from (2) (exactly as in the static condensation of internal nodes of high order FEs), this linear system is reduced to the Schur complement problem

$S x_{\Gamma}=g$,

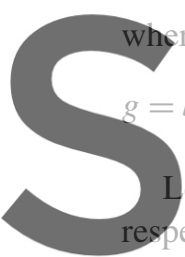
$S=A_{\Gamma \Gamma}-A_{\Gamma I} A_{I I}^{-1} A_{I} \Gamma$, and $=b_{\Gamma}-A_{\Gamma I} A_{I I}^{-1} b_{I}$. Let us denote the cardinalit ectively. The vector space
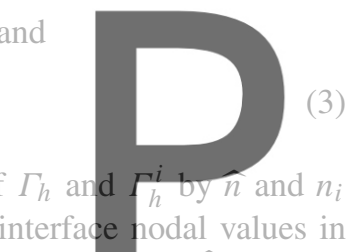
$\Gamma_{h}$ is denoted by $\widehat{V}$; clearly, $\widehat{V}$ is equivalent to $\mathbb{R}^{\widehat{n}}$. We also define the local space $V_{i}$ of interface nodal values on $\Gamma_{l}^{i}$,

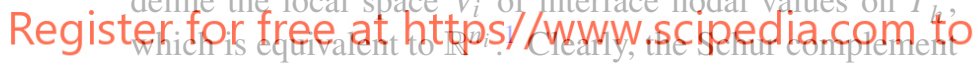
matrix $S: \widehat{V} \times \widehat{V} \rightarrow \mathbb{R}$. System (3) can be written as the assembly (sum) of local Schur complement matrices and right hand side vectors as

$S=\sum_{i=1}^{n_{\mathrm{sbd}}} R_{i}^{t} S_{i} R_{i}, \quad g=\sum_{i=1}^{n_{\mathrm{sbd}}} R_{i}^{t} g_{i}$,

where $R_{i}: \widehat{V} \rightarrow V_{i}$ is the restriction operator and $R_{i}^{t}$ its transpose. The former applied to a vector $y \in \widehat{V}$ gives the vector of local values $y_{i}=R_{i} y \in V_{i}$, while the latter applied to a local vector gives a global vector (filled with zeros for nodes not belonging to subdomain $i$ ). The local Schur complement $S_{i}$ and local right hand side vector $g_{i}$ are defined as:

$$
\begin{aligned}
& S_{i}=A_{\Gamma \Gamma}^{i}-A_{\Gamma I}^{i}\left(A_{I I}^{i}\right)^{-1} A_{I \Gamma}^{i}, \\
& g_{i}=b_{\Gamma}^{i}-A_{\Gamma I}^{i}\left(A_{I I}^{i}\right)^{-1} b_{I}^{i} .
\end{aligned}
$$

\footnotetext{
${ }^{1}$ The spaces $\widehat{V}$ and $V_{i}$ can also be understood in a functional setting as the global and local spaces of discrete harmonic functions (see [6]).
}

The number of subdomains sharing the node with identifier $p$ is denoted by $n(p)$. We will also make use of the global set of replicated local nodes $\Pi_{i=1}^{n_{\mathrm{sbd}}} \Gamma_{h}^{i}$, i.e., $p$ is replicated $n(p)$ times, and the corresponding product space $V=$ $\Pi_{i=1}^{n_{\text {sbd }}} V_{i}$. By definition, the cardinality of this space is $n_{\gamma}=$ $\sum_{i=1}^{n_{\text {sbd }}} n_{i}$, which is equivalent to $\mathbb{R}^{n_{\gamma}} .{ }^{2}$ Any vector $s \in V$ is univocally defined by local values $\left\{s_{i}: i=1, \ldots, n_{\text {sbd }}\right\}$ due to the product space definition. It is possible to obtain an averaged global vector $z \in \widehat{V}$ from $s$ as

$z=\sum_{i=1}^{n_{\text {sbd }}} I_{i} s_{i}$

where $I_{i}=R_{i}^{t} W_{i}: V_{i} \rightarrow \widehat{V}$ is the injection operator, and $W_{i}$ is a diagonal weighting matrix such that

$y=\sum_{i=1}^{n_{\mathrm{sbd}}} I_{i} R_{i} y, \quad$ for any $y \in \widehat{V}$.

$W_{i}$ can be defined as $\left(W_{i}\right)_{p p}=1 / n(p), p=1, \ldots, n_{i}$, although more elaborated expressions must be considered for discontinuous physical properties [42].

We can readily check that $n_{\mathrm{sbd}}=H^{-d}$; assuming a one-

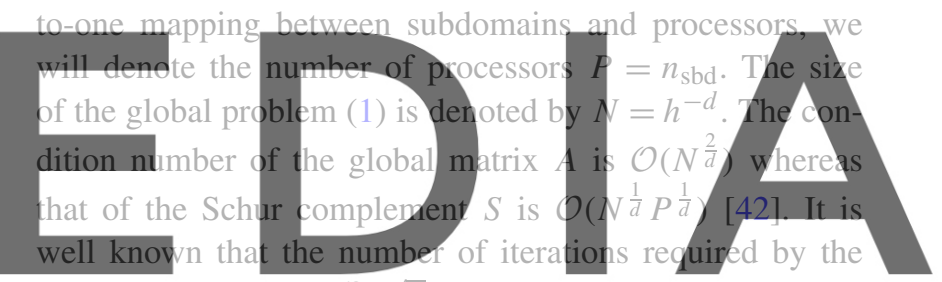

PCG Krylov solver is $\mathcal{O}(\sqrt{\kappa})$, where $\kappa$ is the condition number of the preconditioned.operator [32]. Therefore, the estidownload the version without the watermark

when it is applied to (1) and (3), respectively. Although the number of PCG iterations is certainiy cut down by the restatement of the problem on the interface by the DD approach (since $N \gg P$ for practical ranges of application), there is a lot of margin for improvement via preconditioning. In the rest of this section, we present some non-overlapping DD preconditioners for the Schur complement matrix $S$ such that the resulting condition numbers become (almost) independent of $N$ and $P$.

\subsection{Neumann-Neumann Preconditioner}

We can readily observe that a local contribution to the Schur complement $S_{i}$ is a singular matrix for every floating subdomain $i$, i.e., $\partial \Omega \cap \partial \Omega_{i}=\emptyset$ with $\operatorname{ker}\left(S_{i}\right)=\left\{1_{i}\right\}$ (the space of constant functions). We denote the pseudo-inverse of the

${ }^{2}$ In a functional setting, functions in $\widehat{V}$ are uni-valued on $\Gamma$. On the contrary, since every node $p$ in $\Gamma_{h}$ is replicated $n(p)$ times in $\Pi_{i=1}^{n_{\text {sbd }}} \Gamma_{i}$, functions in $V$ can take different values at different subdomains. As in [42], $\widehat{\bullet}$ is used to denote uni-valued functions on $\Gamma$. 
local Schur complement $S_{i}$ as $S_{i}^{\dagger}$. The Neumann-Neumann (NN) preconditioner is an additive Schwarz preconditioner built from local pseudo-inverses as

$B_{\mathrm{NN}}^{-1}=\sum_{i=1}^{n_{\mathrm{sbd}}} I_{i} S_{i}^{\dagger} I_{i}^{t}$

For non-floating subdomains $S_{i}^{\dagger}=S_{i}^{-1}$ whereas for floating ones $S_{i}^{\dagger}$ applied to a vector $r \in \operatorname{ker}\left(S_{i}\right)^{\perp}$ gives the unique solution of the singular problem $S_{i} x=r$ such that $x \in \operatorname{ker}\left(S_{i}\right)^{\perp}$ (see [39]). When $r \notin \operatorname{ker}\left(S_{i}\right)^{\perp}$, the problem $S_{i} x=r$ does not have a solution and $S_{i}^{\dagger} r$ only minimizes $\left\|r-S_{i} x\right\|$. The following condition number estimate (cf. [42])

$\kappa\left(B_{\mathrm{NN}}^{-1} S\right) \leq C P^{\frac{2}{d}}\left[1+\frac{1}{d^{2}} \log ^{2}\left(\frac{N}{P}\right)\right]$,

gives $\mathcal{O}\left(P^{\frac{1}{d}}\right)$ number of PCG iterations for weak scaling analysis.

2.3 Balancing Neumann-Neumann Preconditioning

The NN preconditioner was enhanced in [28] by introduc-

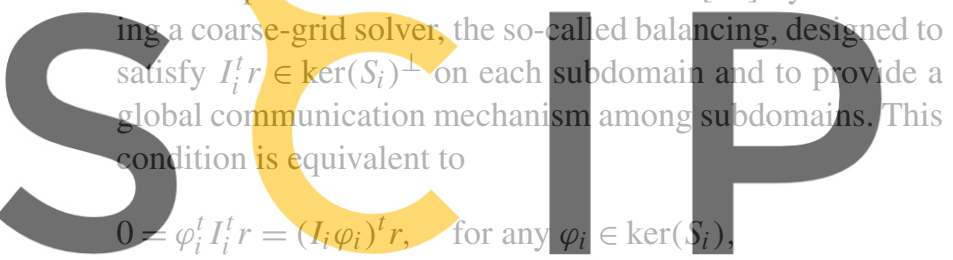

i.e., making the residual orthogonal to the injection $I_{i}$ of

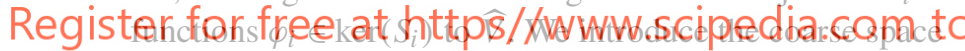

$$
H_{0}=\operatorname{span}\left\{\phi^{i}: i=1, \ldots, n_{\mathrm{sbd}}\right\} \subseteq \widehat{V},
$$

where $\phi^{i}=I_{i} \varphi_{i} ; H_{0}$ is readily represented by $\mathbb{R}^{n_{\mathrm{sbd}}}$ vectors. The coarse-grid preconditioner can be written as

$B_{\mathrm{C}}^{-1}=I_{0} S_{0}^{-1} I_{0}^{t}$,

where $I_{0}: H_{0} \rightarrow \widehat{V}$ is the injection defined as $I_{0} \gamma=$ $\sum_{i=1}^{n_{\text {sbd }}} \phi^{i} \gamma_{i}$ (i.e., the columns of $I_{0}$ are the basis functions $\phi^{i}$ ), and $S_{0}=I_{0}^{t} S I_{0}$ is the coarse-grid space operator.

The coarse-grid balancing preconditioner is combined with the Neumann-Neumann one in a multiplicative fashion, leading to the BNN preconditioner. In order to preserve symmetry, this combination results in the following (naive) form of the BNN preconditioner:

$B_{\mathrm{BNN}}^{-1}=B_{\mathrm{C}}^{-1}+\left(I-B_{\mathrm{C}}^{-1} S\right) B_{\mathrm{NN}}^{-1}\left(I-S B_{\mathrm{C}}^{-1}\right)$.

It can be further shown [41] that if the initial residual in the PCG algorithm is balanced, i.e.,

$r^{*}=\left(I-S B_{\mathrm{C}}^{-1}\right) r$, then the preconditioner can be rewritten as

$B_{\mathrm{BNN}}^{-1}=B_{\mathrm{C}}^{-1}+\left(I-B_{\mathrm{C}}^{-1} S\right) B_{\mathrm{NN}}^{-1}$.

An important observation is that the BNN preconditioner is more efficiently implemented as:

$B_{\mathrm{BNN}}^{-1}=B_{\mathrm{C}}^{-1}\left(I-S B_{\mathrm{NN}}^{-1}\right)+B_{\mathrm{NN}}^{-1}$,

as it was originally proposed in [28]. This equivalent expression results in a three-step application, (1) $z=B_{\mathrm{NN}}^{-1} r$; (2) $t=B_{\mathrm{C}}^{-1}(r-S z)$; (3) update $z:=z+t$. Let us remark that in this case the application of the BNN preconditioner only requires to solve one coarse-grid problem. A modified implementation of the algorithm that leads to a spare of one Dirichlet solve per PCG iteration has recently been proposed in [2]. ${ }^{3}$ The condition number estimate (cf. [28])

$\kappa\left(B_{\mathrm{BNN}}^{-1} S\right) \leq C\left[1+\frac{1}{d^{2}} \log ^{2}\left(\frac{N}{P}\right)\right]$.

results in a constant number of PCG iterations for weak scaling analysis.

\subsection{Balancing DD by Constraints Preconditioner}

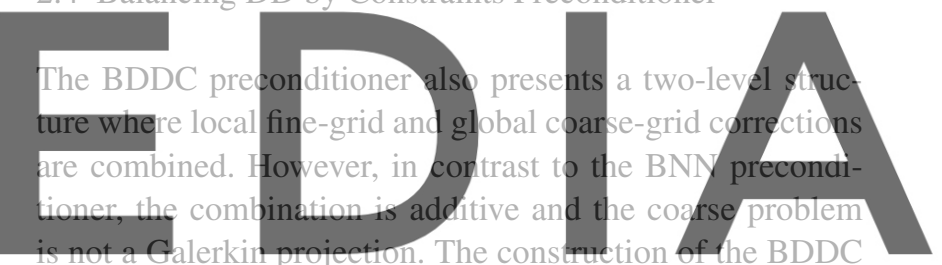

preconditioner is based on a topological classification of the

\section{dordo
faces}

We denote by $N(p)$ the index set of subdomains that share node $p$, i.e., $N(p)=\left\{i: p \in \Gamma_{h}^{i}\right\}$, with cardinality already defined in Sect. 2.1 as $n(p)$. We can construct the set $\mathcal{G}=\left\{G_{a}: a=1, \ldots, n^{\text {cts }}\right\}$, where every object $G_{a}$ is a maximal subset of nodes in $\Gamma_{h}$ with identical index set, i.e., $N(p)=N(q)$ for any $p, q \in G_{a}$, denoted as $N\left(G_{a}\right) .{ }^{4}$ Now, we can consider a topological classification of the objects as follows: $G_{a}$ is a face if $\left|N\left(G_{a}\right)\right|=2$ and $\left|G_{a}\right|>1$, an edge if $\left|N\left(G_{a}\right)\right|>2$ and $\left|G_{a}\right|>1$ and a corner if $\left|G_{a}\right|=1$; this definition corresponds to a 3D space but can readily be restricted to 2D. Grouping together the objects of the same type, we obtain the set of faces $\mathcal{F}=\left\{F_{a}, a=1, \ldots, n^{F}\right\}$,

\footnotetext{
${ }^{3}$ It is based on the observation that $S I_{0} v_{0}$ for $v_{0} \in H_{0}$ can readily be obtained as a linear combination of $S \phi^{i}$ quantities, that have already been computed when assembling $S_{0}$ at the preconditioner set-up. This observation, combined with a slight modification of the PCG recurrence described in [2], spares one Schur complement-vector product per PCG iteration.

${ }^{4}$ This definition of $\left\{G_{a}: a=1, \ldots, n_{c t s}\right\}$ generates a unique partition of $\Gamma_{h}$.
} 
the sets of edges $\mathcal{E}=\left\{E_{a}, a=1, \ldots, n^{E}\right\}$ and the set of corners $\mathcal{C}=\left\{C_{a}, a=1, \ldots, n^{C}\right\}$; clearly $n_{\mathrm{cts}}=n_{F}+n_{E}+n_{C}$. We can consider the restriction of all these sets to a given subdomain $i$ as follows: we define $\mathcal{G}_{i}=\left\{G_{a} \in \mathcal{G}: i \in\right.$ $\left.N\left(G_{a}\right)\right\}$ and $n_{\text {cts }}^{i}=\left|\mathcal{G}_{i}\right|$ denotes the number of constraints on subdomain $i$; analogously for $\left(\mathcal{F}_{i}, n_{F}^{i}\right),\left(\mathcal{E}_{i}, n_{E}^{i}\right)$ and $\left(\mathcal{C}_{i}, n_{C}^{i}\right)$.

The local, fine-grid preconditioner in the BDDC method is defined as

$B_{\mathrm{F}}^{-1}=\sum_{i=1}^{n_{\mathrm{sbd}}} I_{i}\left(S_{i}^{c}\right)^{-1} I_{i}^{t}$,

where $\left(S_{i}^{c}\right)^{-1}$ is a "constrained" inverse of the local Schur complement $S_{i}$. The application of $\left(S_{i}^{c}\right)^{-1}$ to a vector $r$ involves the solution of the following (constrained) linear system

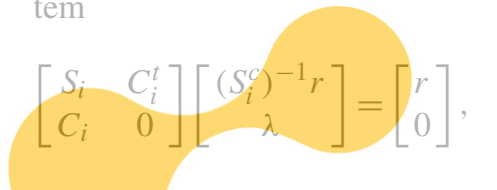

where $C_{i} \in R^{n_{\mathrm{cts}}^{i} \times n_{i}}$ is the matrix of constraints. Denoting by $l_{i}(a)$ the local numbering of $G_{a} \in \mathcal{G}_{i}$ at subdomain $i$, the


norm. With such a definition, the product $z=C_{i} y$, with $z \in$ $R^{n^{i} i_{\text {cts }}}$ and $y \in R^{n_{i}}$ gives a vector $y$ whose component $y_{l_{i}(q)}$

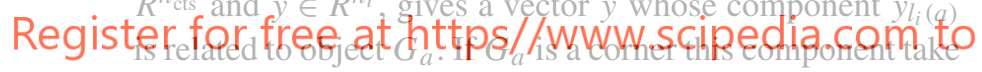
the value of $z$ in this comer, i.e., $y_{l_{i}(a)}=z_{l_{i}(a)}$; if $G_{a}$ is a face (edge), it takes the mean value of $z$ on the face (edge), i.e., $z_{l_{i}(a)}=\left|G_{a}\right|^{-1} \sum_{j \in G_{a}} y_{j}$. The three most common variants of the BDDC method, here referred as BDDC(c), BDDC(ce) and BDDC(cef), are based on only corner constraints, corner and edge constraints, and corner, edge and face constraints, respectively.

The coarse space $H_{0} \subseteq V$ is defined as

$$
H_{0}=\operatorname{span}\left\{\phi^{a}: a=1, \ldots, n_{\mathrm{cts}}\right\}
$$

where every coarse function $\phi^{a}$ is associated with coarse object $G_{a}$. Coarse functions are constructed as the tensor product of local values, i.e., $\phi^{a}=\left\{\phi_{i}^{a}, i=1, \ldots, n_{\text {sbd }}\right\} \in V$. If $i \in N\left(G_{a}\right), \phi_{i}^{a}$ is obtained as the solution of

$$
\left[\begin{array}{cc}
S_{i} & C_{i}^{t} \\
C_{i} & 0
\end{array}\right]\left[\begin{array}{c}
\phi_{i}^{a} \\
\lambda
\end{array}\right]=\left[\begin{array}{c}
0 \\
e_{l_{i}(a)}
\end{array}\right]
$$

where $e_{b}$ denotes the $b$ column of the identity matrix; $\phi_{i}^{a}=$ 0 otherwise. Note that by its definition the coarse space is non-conforming, i.e., $H_{0} \not \subset \widehat{V}$. The coarse-grid space basis functions define the mapping $I_{0}: H_{0} \rightarrow \widehat{V}$ as

$I_{0} \gamma=\sum_{a=1}^{n_{\mathrm{cts}}} \sum_{i=1}^{n_{\mathrm{sbd}}} I_{i} \phi_{i}^{a} \gamma_{a}$,

and the coarse space operator as

$\left(S_{0}\right)_{a b}=\sum_{i=1}^{n_{\mathrm{sbd}}} R_{i}^{t} \phi_{i}^{a} S_{i} \phi_{i}^{b} R_{i}$.

The final preconditioner can be written as an additive combination of a coarse and a fine-grid contribution

$B_{\mathrm{BDDC}}^{-1}=B_{\mathrm{C}}^{-1}+B_{\mathrm{F}}^{-1}$,

where $B_{\mathrm{C}}^{-1}=I_{0} S_{0}^{-1} I_{0}^{t}$ as in the BNN method. The condition number estimate (cf. [29])

$\kappa\left(B_{\mathrm{BDDC}}^{-1} S\right) \leq C\left[1+\frac{1}{d^{2}} \log ^{2}\left(\frac{N}{P}\right)\right]$,

results in a constant number of PCG iterations (neglecting the logarithmic factor) for fixed load per core and increasing number of cores. For the 2D Poisson problem, BDDC(c) al-

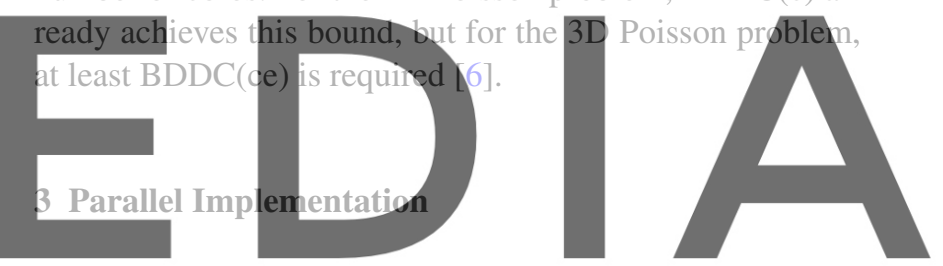

In this section we describe in detail a parallel distributed-

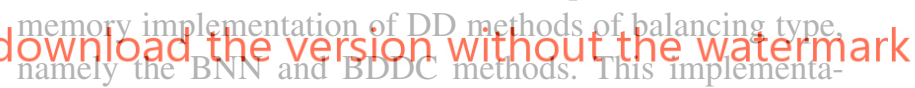

tion inherits the two-level structure of the preconditioners subject of study. On the first level, the subdomains resulting from the non-overlapping partition of the global mesh are mapped to the MPI tasks, with a one-to-one mapping among subdomains, MPI tasks and computational cores of the underlying distributed-memory computer. On this level, all data structures required for the (preconditioned) iterative solution of the interface problem (3) are distributed among MPI tasks conformally with the underlying non-overlapping partition, and both computation and message-passing among MPI tasks are inherently of local nature, therefore, highly parallel. On the second level, the one corresponding to the global coupling among subdomains, the coarse-grid problem is assembled and solved serially on one processor (or all processors in the communicator) and therefore no parallelism is exploited at all.

For the sake of efficiency and portability, our implementation relies on several standard computational kernels provided by the dense/sparse BLAS and LAPACK, and highly efficient cache-aware vendor implementations of these kernels in order to achieve high flop rates on the computational 
core level (such as Intel MKL or IBM ESSL for Intel and IBM PPC multicore CPUs, respectively). Besides, through proper interfaces to third party libraries (e.g., PARDISO [36, 37]), the local Dirichlet (Schur complement-vector product) and Neumann (fine-grid correction) problems, as well as the global coarse-grid problem, are solved via cuttingedge sparse direct solvers. These solvers typically follow a super-nodal/multi-frontal approach for the efficient exploitation of the core cache subsystem thorough the level 3 BLAS [8]. The use of sparse direct methods is mandatory in the current setting as the iterative solution of (3) requires the Schur complement-vector to be computed exactly. ${ }^{5}$ Besides, sparse direct solvers are highly robust numerically and very successful in the efficient exploitation of the cache hierarchy. The former factor is very helpful for the validation of computer implementations, while the latter one is becoming more important given the poor (main memory) bandwidth scalability in current/future multi-core/many-core CPUs.

The implementation is (essentially) split into two main phases. The first phase sets up the Schur complement and preconditioner before starting the iterations. This phase is in turn divided into a symbolic phase, where the geometrical information is computed beforehand (e.g., the sparsity pat-
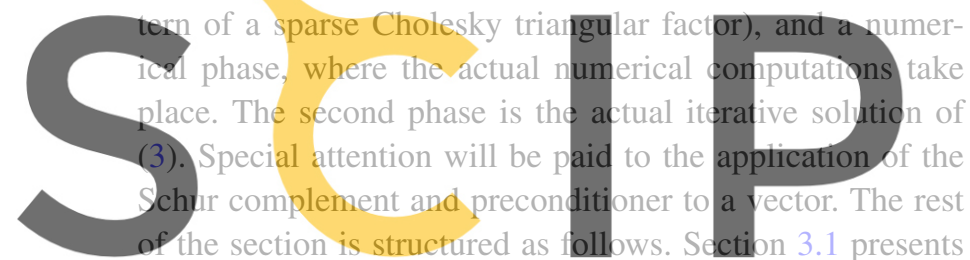

the data distribution and the general setting for a distributed-

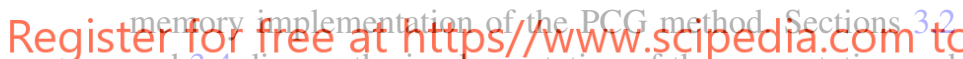
and 3.4 discuss the implementation of the computation and communication kernels required by the fine-grid preconditioning level, respectively, while Sects. 3.3 and 3.5 focus on those required by the coarse-grid preconditioning level.

\subsection{Data Distribution and Basic Building Blocks}

Algorithm 1 depicts the BDD-PCG iterative solver applied to the interface problem (3). ${ }^{6}$ In a distributed-memory implementation of this algorithm, all vectors $y \in \widehat{V}$ and the Schur complement $S \in \widehat{V} \otimes \widehat{V}$ are partitioned and distributed

\footnotetext{
${ }^{5} \mathrm{BDD}$ methods can certainly be reformulated as preconditioners for the global linear system (1). This enables approximate solvers (e.g., AMG $[35,40,43])$ to be used in conjunction with BDD methods. Although this approach relaxes the arithmetic/memory demands of sparse direct solvers (particularly in 3D), it would result in a new source of difficulties (e.g., robustness/complexity trade-off evaluation, parameter tuning, load unbalancing issues, poor flop rates) that deserve further research.

${ }^{6}$ For the BNN method, we assume that the initial value $x_{0}$ is such that $r_{0}$ is balanced (see [28]).
}

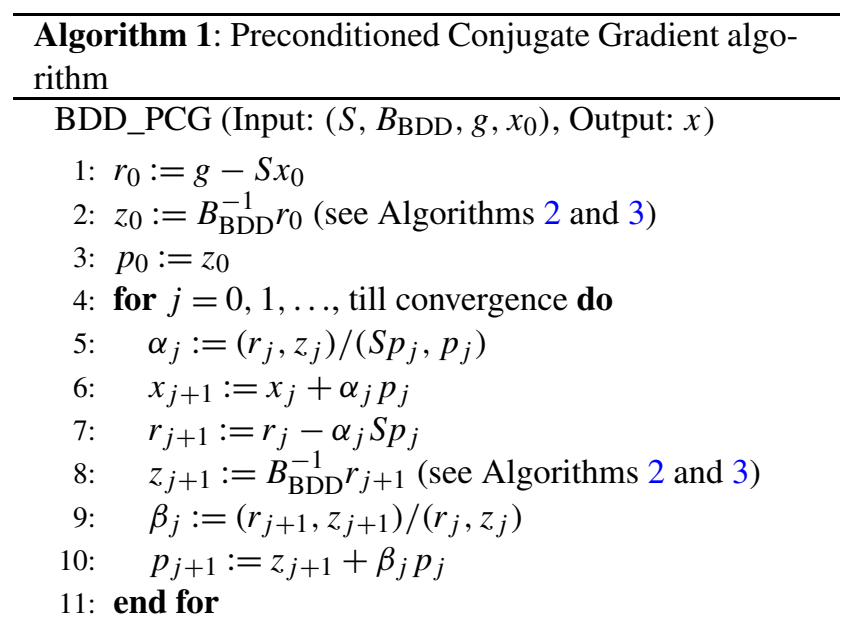

among MPI tasks conformally with the underlying nonoverlapping partition of the global interface. For those vectors $y \in \widehat{V}$ in Algorithm 1 which are naturally expressed as the assembly (sum) of subdomain contributions (in particular $r, A p, g$ ), it is convenient (for reasons made clear below) that each MPI task keeps on its local address space partially

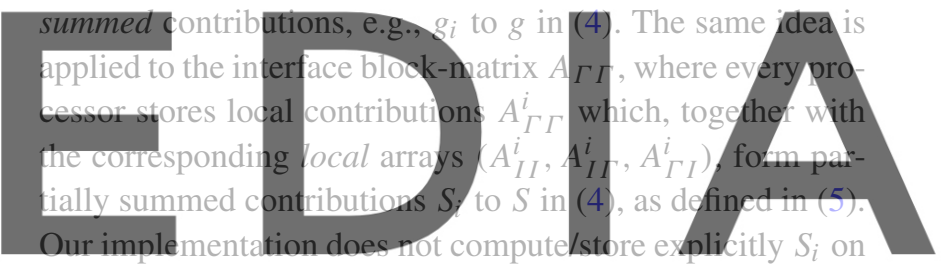
each MPI task. Instead, the application of $S$ to a vector is

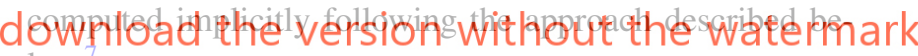 low.}

On the other hand, for the rest of vectors $y \in \widehat{V}$ in Algorithm 3 (in particular, $x, z, p$ ), it is convenient that each MPI task keeps local fully summed (i.e., assembled) entries $y_{i}$, such that $y_{i}=R_{i} y$. Finally, any vector in the product space $v \in V$ is naturally distributed in such a way that each MPI task stores one local component $v_{i} \in V_{i}$ of $v$. Vectors $v \in V$ are not explicitly present in Algorithm 1, but as the result of intermediate steps during the application of the preconditioner. Following this approach, Algorithm 1 is therefore implemented in a subdomain-by-subdomain form, in the same way as in element-by-element techniques [13].

We next enumerate the basic building blocks of Algorithm 1, highlighting implementation details in our distributed-memory codes:

\footnotetext{
${ }^{7}$ An explicit assembly of $S_{i}$ is required for some DD preconditioners (see e.g. [17]). Besides, this approach allows one to exploit the dense level 2 BLAS for the Schur complement-vector product, which can only compensate the expensive set-up of $S_{i}$ for non-scalable preconditioners with high iteration counts.
} 

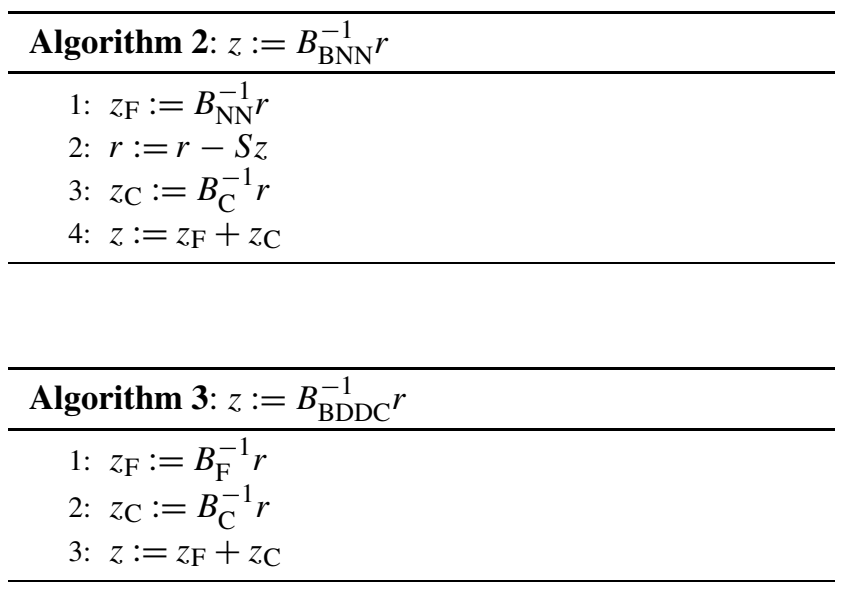

Task 1 Vector updates in lines 6, 7 and 10. No communications are required. Each MPI task performs a local update using local data structures.

Task 2 Computation of inner products in lines 5 and 9. The computation of the inner product $z^{t} r$ is particularly simple as

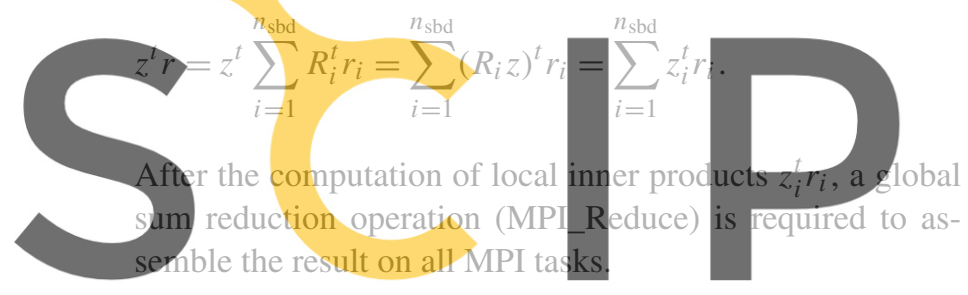

Register for for freation of the Schur complement operator in forms a local product $g_{i}=S_{i} y_{i}$. This local operation results in a vector $g$ such that each MPI task keeps local partially summed contributions, i.e.:

$g=S y=\sum_{i=1}^{n_{\mathrm{sbd}}} R_{i}^{t} S_{i} R_{i} y=\sum_{i=1}^{n_{\mathrm{sbd}}} R_{i}^{t} S_{i} y_{i}=\sum_{i=1}^{n_{\mathrm{sbd}}} R_{i}^{t} g_{i}$.

During Schur complement set-up, $A_{I I}^{i}$ is extracted from $A^{i}$ and the sparse Cholesky factorization of $A_{I I}^{i}$ is computed. The product $S_{i} y_{i}$ is computed following a three-step algorithm: (1) compute $t=-A_{I \Gamma}^{i} y_{i}$;(2) solve $A_{I I}^{i} u=t$; (3) $g_{i}=A_{\Gamma \Gamma}^{i} y_{i}+A_{\Gamma I}^{i} u$. A sparse backward/forward substitution is required for step (2), and one and two sparse matrixvector products for steps (1) and (3), respectively. These sparse matrix-vector products are performed using a standard (sparse) level 2 BLAS kernel.

Task 4 The application of the preconditioner in lines 2 and 8 as shown in Algorithms 2 and 3 for the BNN and BDDC preconditioners, respectively. Here is where the twolevel structure of both preconditioners is exposed. In the case of the BNN method, there is an additional residual update; see line 2 in Algorithm 2. The Schur complementvector in this update is performed as in (13).

The first step in the application of the preconditioner is the computation of the fine-grid correction $z_{\mathrm{F}}$ (see line 1 in Algorithms 2 and 3). For both methods, the fine-grid preconditioner is applied to a global vector $r$ (a residual) which is distributed in such a way that each MPI task keeps local partially summed contributions. In both the application of $B_{N N}^{-1}$ and $B_{\mathrm{F}}^{-1}$, the following computation has to be performed first:

$I_{i}^{t} r=I_{i}^{t} \sum_{j=1}^{n_{\mathrm{sbd}}} R_{j}^{t} r_{j}$

which requires to obtain fully summed entries of $r$ at each MPI task, followed by the application of the weighting matrix. The former fully summed assembly of $r$ involves communication among nearest neighbors. The efficient implementation of this communication kernel is described in Sect. 3.4. The latter application of the weighting matrix is highly parallel as it can be applied locally on each MPI task. After the solution of local fine-grid problems (see Sect. 3.2), we obtain $s_{i}=S_{i}^{\dagger} I_{i}^{t} r$ and $s_{i}=\left(S_{i}^{c}\right)^{-1} I_{i}^{t} r$ for the BNN and BDDC methods, respectively, which define a global element
of the product space $s_{\mathrm{F}} \in X$. Finally, it is transforned to a
continuous $z_{\mathrm{F}} \in \widehat{V}$ as in (6). This operation also involves
communication among nearest neighbors, as described in
Sect. 3.4.
The second step in the application of the preeorditioner is the computation of the coarse-grid correction $z_{\mathrm{C}}$ (see lines 3

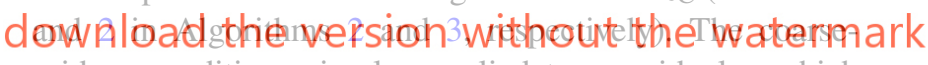
grid preconditioner is also applied to a residual $r$ which is distributed in such a way that each MPI task stores partially summed contributions. For both preconditioners the first step is a projection onto the coarse space. For the BNN method it reads as:

$$
\left(I_{0}^{t} r\right)_{i}=\left(\phi^{i}\right)^{t} r=\left(\phi^{i}\right)^{t} \sum_{j \in N(i)} R_{j}^{t} r_{j}=\sum_{j \in N(i)}\left(R_{j} \phi^{i}\right)^{t} r_{j}
$$

where abusing the notation of Sect. 2.3, N(i) is the set of subdomains neighboring subdomain $i$ (included itself). For the BDDC method, since the coarse-grid space is non-conforming (see (11)), we compute $\left(I_{0}^{t} r\right)_{a}=\sum_{i \in N(a)}\left(\phi_{i}^{a}\right)^{t} I_{i}^{t} r$, where the computation of $I_{i}^{t} r$ is reused from the fine-grid component (see (14)). The solution of the global problem $\gamma=S_{0}^{-1} I_{0}^{t} r \in H_{0}$ is then injected into the fine-grid space. For the BNN method, since $H_{0} \subset \widehat{V}$ and $z_{\mathrm{C}}=$ $I_{0} \gamma=\sum_{i=1}^{n_{\mathrm{sbd}}} \phi^{i} \gamma_{i}$, we can compute locally $\left(z_{\mathrm{C}}\right)_{i}=R_{i} z_{\mathrm{C}}=$ $\sum_{j \in N(i)} R_{i} \phi^{j} \gamma_{j}$. In the case of the BDDC method, we first compute the local components $\sum_{a \in \mathcal{G}_{i}} \phi_{i}^{a} \gamma_{a}$ of $s_{\mathrm{C}} \in V$ which 
has to be transformed to a continuous $z_{\mathrm{C}} \in \widehat{V}$ as described above with $z_{\mathrm{F}}$. Note that as both the fine-grid and coarsegrid correction lead to $s_{\mathrm{F}} \in V$ and $s_{\mathrm{C}} \in V$, respectively, we can actually compute $s=s_{\mathrm{F}}+s_{\mathrm{C}}$ in line 3 of Algorithm 3, to finally obtain $z$ from $s$ as in (6). In other words, the above communication required to transform $s_{\mathrm{F}} \in V$ to a continuous $z_{\mathrm{F}} \in \widehat{V}$ can be postponed until the coarse-grid and fine-grid corrections are combined. The distributed-memory implementation of $I_{0}^{t} r$ and $I_{0} \gamma$ requires global communications as described in Sect. 3.5. Besides, they require the coarse-grid basis functions which are obtained as the solution of local problems as described in Sect. 3.3. The assembly and solution of the coarse-grid problem is also described in Sect. 3.3.

As it is apparent from the description of Algorithm 1, the distributed-memory implementation of BDDC and BNN methods has only subtle differences, so that a common implementation framework for both has been used.

\subsection{Fine-Grid Preconditioning Level}

This section describes the computations to be performed in the fine-grid preconditioning level of the BNN (Sect. 3.2.1) and BDDC (Sect. 3.2.2) preconditioners. Special emphasis is put on the identification of the (dense/sparse) standard

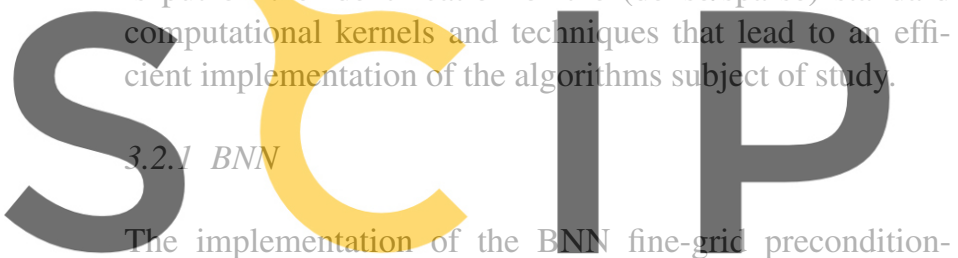

ing level must take care of the efficient computation of

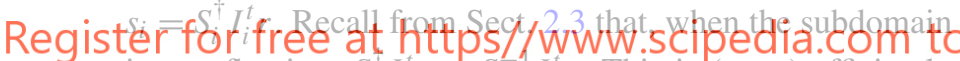
is non-floating, $S_{i}^{t} I_{i}^{t} r=S_{i}^{-1} I_{i}^{t} r$. This is (most) efficiently computed as the solution of the following linear system:

$\left[\begin{array}{ll}A_{I I}^{i} & A_{I \Gamma}^{i} \\ A_{\Gamma I}^{i} & A_{\Gamma \Gamma}^{i}\end{array}\right]\left[\begin{array}{c}t \\ s_{i}\end{array}\right]=\left[\begin{array}{c}0 \\ I_{i}^{t} r\end{array}\right]$

On the other hand, when the subdomain is floating (i.e., $S_{i}$ is singular), then $S_{i}^{\dagger} I_{i}^{t} r$ is computed as the solution of the following (constrained) local system:

$$
\left[\begin{array}{ccc}
A_{I I}^{i} & A_{I \Gamma}^{i} & 0 \\
A_{\Gamma I}^{i} & A_{\Gamma \Gamma}^{i} & 1_{i} \\
0 & 1_{i}^{t} & 0
\end{array}\right]\left[\begin{array}{c}
t \\
s_{i} \\
\lambda
\end{array}\right]=\left[\begin{array}{c}
0 \\
I_{i}^{t} r \\
0
\end{array}\right],
$$

where $\lambda \in \mathbb{R}$ is the Lagrange multiplier, with $\lambda=0$ as $I_{i}^{t} r \in \operatorname{ker}\left(S_{i}\right)^{\perp}=\operatorname{span}\left(1_{i}\right)^{\perp}$ by construction of the BNN coarse-grid space (see Sect. 2.3). We stress that the constrained linear system (17) is symmetric indefinite but nonsingular. Although $\lambda$ is known in advance, the elimination of the third equation in (17) leads to a singular problem, therefore unsolvable by sparse direct solvers (this is indeed one of the main drawbacks of some BNN implementations).
The solution of (16) and (17) requires, during preconditioner set-up, the computation of a sparse direct factorization of their corresponding coefficient matrix, while the application of the preconditioner requires a sparse backward/forward substitution to finally obtain $s_{i}$. The algorithms for the direct solution of symmetric indefinite linear systems (e.g. (17)) are typically more expensive than those required for symmetric definite ones (e.g. (16)). ${ }^{8}$ An alternative approach (also implemented in our codes) that we recommend to deal with floating subdomains is the one presented in [2]. Essentially, it is based on the observation that (17) can be transformed into an equivalent positive definite (PD) system by simply fixing judiciously (in particular by analyzing the kernel of $S_{i}$ ) picked degrees of freedom; for the Laplacian problem it simply reduces to fix one arbitrary degree of freedom. We refer the reader to $[2]$ for a detailed explanation and comparison of both approaches for elasticity and Laplacian problems.

\subsubsection{BDDC}

The BDDC fine-grid preconditioning level is responsible for the computation of the product $s_{i}=\left(S_{i}^{c}\right)^{-1} I_{i}^{t} r$. This is ob-
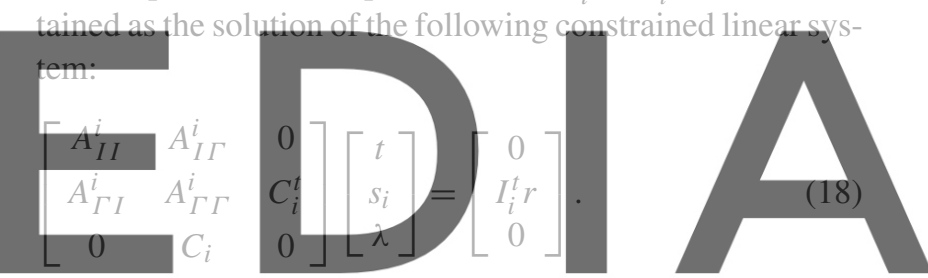

There are two solution approaches for this symmetric in-

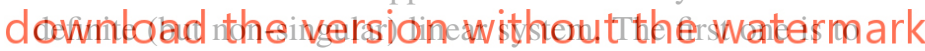
tackle (18) directly using a sparse direct solver for symmetric indefinite linear systems. In such a case, a sparse direct factorization of the coefficient matrix is computed for the set-up of the preconditioner, while a sparse backward/forward substitution is required to compute $s_{i}$ during preconditioner application. The second approach, originally presented in [10], exploits the particular structure of $C_{i}$ to enable the exploitation of symmetric definite problems for the solution of (18). In the rest of this section we discuss this second approach and its efficient implementation.

Let us consider a reordering of (18) in such a way that FE equations/unknowns related to corners (C) are numbered first, followed by the rest $(\mathrm{R})$ of nodes, ordered as internal nodes first, followed by nodes members of faces and nodes

\footnotetext{
${ }^{8}$ For example, PARDISO is based on the sparse Cholesky factorization without pivoting for symmetric positive definite problems, while for symmetric indefinite problems, it uses a more expensive sparse $L D L^{T}$ factorization which, for numerical stability purposes, combines static (prior-to-factorization) pivoting via symmetric weighted matchings and classical Bunch-Kaufman dynamic (during factorization) pivoting only applied inside the supernodes [36, 37].
} 
members of edges. Further, rows and columns of $C_{i}$ related to nodes in $\mathcal{C}_{i}$ are labeled first, followed by those of $\mathcal{F}_{i}$ and $\mathcal{E}_{i}$. Let us also assume that the local ordering of corners is conformal with that of the rows of $C_{i}$ corresponding to corner constraints. Then we obtain the following block reordered (constrained) linear system:

$$
\left[\begin{array}{cccc}
A_{C C}^{i} & \left(A_{R C}^{i}\right)^{t} & I & 0 \\
A_{R C}^{i} & A_{R R}^{i} & 0 & \left(C_{R}^{i}\right)^{t} \\
I & 0 & 0 & 0 \\
0 & C_{R}^{i} & 0 & 0
\end{array}\right]\left[\begin{array}{c}
x_{C}^{i} \\
x_{R}^{i} \\
\lambda_{C}^{i} \\
\lambda_{R}^{i}
\end{array}\right]=\left[\begin{array}{c}
b_{C}^{i} \\
b_{R}^{i} \\
0 \\
0
\end{array}\right],
$$

where

$$
\begin{aligned}
& A_{R R}^{i}=\left[\begin{array}{ccc}
A_{I I}^{i} & A_{I F}^{i} & A_{I E}^{i} \\
A_{F I}^{i} & A_{F F}^{i} & A_{F E}^{i} \\
A_{E I}^{i} & A_{E F}^{i} & A_{E E}^{i}
\end{array}\right] \\
& C_{R}^{i}=\left[\begin{array}{lll}
0 & C_{F}^{i} & 0 \\
0 & 0 & C_{E}^{i}
\end{array}\right],
\end{aligned}
$$

and $\lambda_{C}^{i}$ and $\lambda_{R}^{i}$ are the Lagrange multipliers associated to corner constraints and the rest of constraints, respectively.

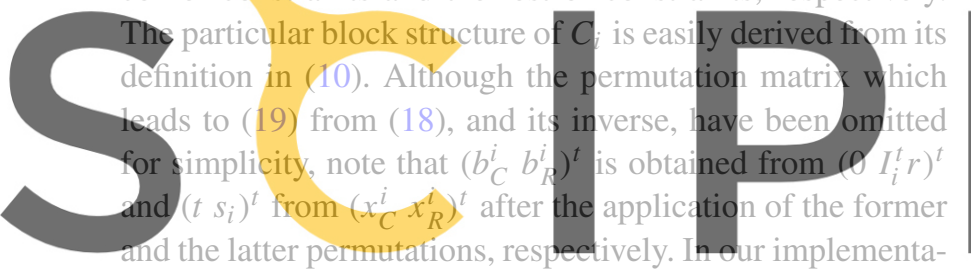
tion, both permutations are explicitly computed (and stored

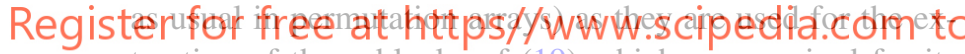
traction of those blocks of (19) which are required for its solution (see below).

The solution of (19) is computed as follows. From the third and second block equations of (19) we have that $x_{C}^{i}=$ 0 and

$x_{R}^{i}=\left(A_{R R}^{i}\right)^{-1} b_{R}^{i}-\left(A_{R R}^{i}\right)^{-1}\left(C_{R}^{i}\right)^{t} \lambda_{R}^{i}$,

respectively, where $A_{R R}^{i}$ is a large, sparse, symmetric positive definite matrix. If the first two block equations are eliminated from (19), Lagrange multipliers $\lambda_{R}^{i}$ are obtained as the solution of the following system:

$C_{R}^{i}\left(A_{R R}^{i}\right)^{-1}\left(C_{R}^{i}\right)^{t} \lambda_{R}^{i}=-\left(C_{R}^{i}\right)^{t}\left(A_{R R}^{i}\right)^{-1} b_{R}^{i}$,

where $C_{R}^{i}\left(A_{R R}^{i}\right)^{-1}\left(C_{R}^{i}\right)^{t}$, the Schur complement matrix associated to $\lambda_{R}^{i}$, is a small, dense, symmetric positive definite matrix (of size $n_{i}^{F}+n_{i}^{C}$ ). During preconditioner set-up, the following four tasks are performed: (1) compute the sparse Cholesky factorization of $A_{R R}^{i} ;(2)$ compute $\left(A_{R R}^{i}\right)^{-1}\left(C_{R}^{i}\right)^{t}$ by means of a kernel which allows the exploitation of the level 3 BLAS during the (blocked) sparse backward/forward substitution, and store the result in a dense work array for later use; (3) compute $C_{R}^{i}\left(A_{R R}^{i}\right)^{-1}\left(C_{R}^{i}\right)^{t}$ (reusing $\left(A_{R R}^{i}\right)^{-1}\left(C_{R}^{i}\right)^{t}$ from step (2)); (4) compute a dense Cholesky factorization of $C_{R}^{i}\left(A_{R R}^{i}\right)^{-1}\left(C_{R}^{i}\right)^{t}$ using the corresponding LAPACK kernel. We stress that $\left(C_{R}^{i}\right)^{t}$ is stored in dense storage mode as required by the kernel exploited in step (2). However, $C_{R}^{i}$ is not stored. Instead, it is more efficient to implement the matrix-vector (and matrix-matrix) multiplication using a subroutine which generates its entries "on the fly" to save storage and that only operates with the non-zero entries of $C_{R}^{i}$ to save floating-point calculations. On the other hand, during the preconditioner application, the following tasks are performed: (1) solve $A_{R R}^{i} t_{R}^{i}=$ $b_{i}^{R}$ by sparse backward/forward substitution; (2) compute $w_{R}^{i}=C_{R}^{i} t_{R}^{i}$; (3) solve $C_{R}^{i}\left(A_{R R}^{i}\right)^{-1}\left(C_{R}^{i}\right)^{t} \lambda_{R}^{i}=-w_{R}^{i}$ using the corresponding level 2 BIAS kernel for the triangular solution of dense linear systems; (4) compute $x_{R}^{i}=$ $t_{R}^{i}-\left(A_{R R}^{i}\right)^{-1}\left(C_{R}^{i}\right)^{t} \lambda_{R}^{i}$. The computation of step (4) requires a former level 2 BLAS dense matrix-vector product. The dense work array setup during preconditioner construction for the storage of $\left(A_{R R}^{i}\right)^{-1}\left(C_{R}^{i}\right)^{t}$ is reused for this product.

\subsection{Coarse-Grid Preconditioning Level}

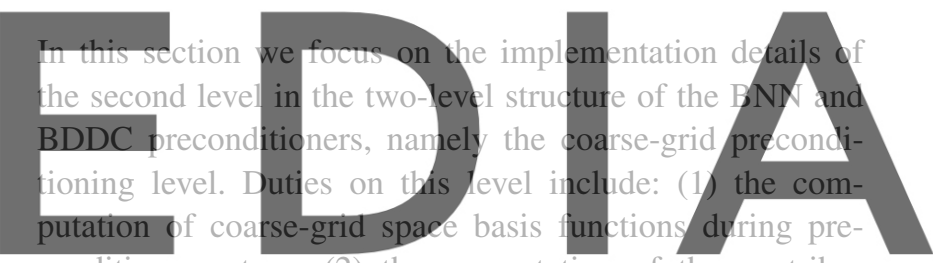

conditioner set-up; (2) the computation of the contribution of each subdomain to the coarse-grid coefficient ma-

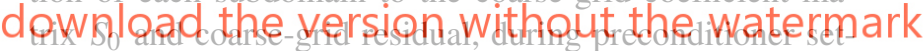

up and application, respectively; (3) the assembly, Cholesky factorization of $S_{0}$ and solution of the coarse-grid system during preconditioner set-up and application, respectively. Although there are several implementation approaches for (3) in a distributed-memory code (e.g., in parallel on all or a subset of processors in the global communicator), we turn our attention into a MPI implementation that solves the coarse-grid problem serially. Apart from the evaluation of the scalability of this solution, another purpose of this paper is to determine whether it is more efficient to assemble/factorize/solve the coarse-grid problem on one processor, and then distribute the solution over the rest of processors, or to assemble/factorize/solve an identical problem on all processors, where no global communication is required afterwards. The solution on one or all processors decision depends on the extra synchronization and communication overhead incurred by the global collectives required to implement each option. Section 3.5 presents these collectives and evaluates their performance and scalability on a largescale distributed-memory machine, and Sect. 4 studies the weak scalability of the coarse-grid preconditioning level, 
paying special attention to the one or all processors decision. The rest of the current section describes in detail implementation considerations of this preconditioning level.

We denote by $\Phi_{i}$ the matrix whose columns are the local coarse basis functions. In the case of the BNN method they are $R_{i} \phi^{j}$ for $j \in N(i)$. In the case of the BDDC method they are $\phi_{i}^{a}$ for $a \in \mathcal{G}_{i}$ (see Sect. 2.4). In the case of the BDDC method, the computation of $\Phi_{i}$ involves the solution of the following (constrained) sparse linear system with several right hand sides:

$$
\left[\begin{array}{ccc}
A_{I I}^{i} & A_{I \Gamma}^{i} & 0 \\
A_{\Gamma I}^{i} & A_{\Gamma \Gamma}^{i} & C_{i}^{t} \\
0 & C_{i} & 0
\end{array}\right]\left[\begin{array}{c}
\beta_{i} \\
\Phi_{i} \\
\Lambda_{i}
\end{array}\right]=\left[\begin{array}{l}
0 \\
0 \\
I
\end{array}\right],
$$

where $\phi_{i}^{a}$, i.e., the a-column of $\Phi$, is the restriction of a (corner, edge, or face) constraint basis function to subdomain $i, \beta_{i}^{j}$ is the discrete harmonic extension of $\phi_{i}^{j}, \lambda_{i}^{j}$ is the vector of Lagrange multipliers, and $I$ is the identity matrix of size $n_{\text {cts. }}^{i}$. The solution of (22) reuses the data structures which are computed during the set-up of the fine-grid preconditioner; see Sect. 3.2.2. Any of the two approaches described for the solution of (18) can be used for (22). How-

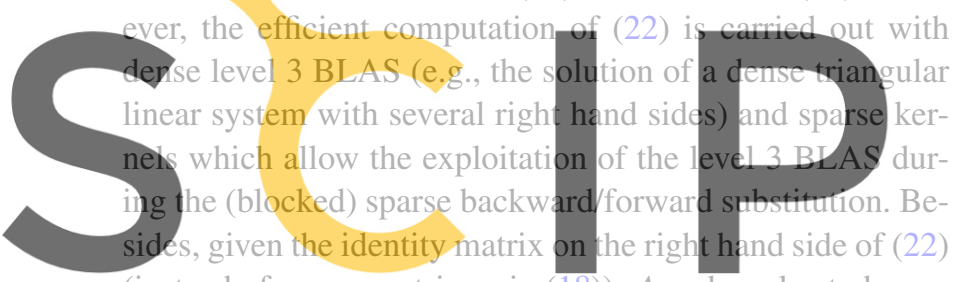
(instead of a zero matrix as in (18)), $A_{R C}$ has also to be extracted from (19) during preconditioner set-up and stored as

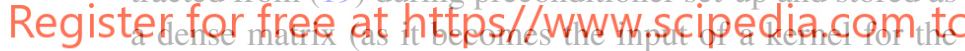
solution of triangular systems with several right hand sides). Coarse-grid preconditioning level duties also include the computation of the contribution of each subdomain to the coarse-grid coefficient matrix $S_{0}$ and coarse-grid residual. The sparse matrix $S_{0}$ can be obtained as the assembly (sum) of subdomain contributions $\sum_{i=1}^{n_{\text {sbd }}}\left(\Phi_{i}\right)^{t} S_{i} \Phi_{i}$ as in (12). In the case of the BNN method, the computation of $S_{i} \Phi_{i}$ is split (as in (13)) into two steps: (1) solve $A_{I I}^{i} \beta_{i}=-A_{I \Gamma}^{i} \Phi_{i}$; (2) $S_{i} \Phi_{i}=A_{\Gamma I}^{i} \beta_{i}+A_{\Gamma \Gamma}^{i} \Phi_{i}$. Step (1) is efficiently computed with a kernel for the sparse direct solution of linear systems with several right hand sides, i.e., a kernel which allows the exploitation of the level 3 BLAS during the (blocked) sparse backward/forward substitution. Step (2) requires a pair of sparse-dense matrix-matrix multiplications, which are efficiently computed using the corresponding kernel in the (sparse) level 3 BLAS. In case of the BDDC method, the computation of $S_{i} \Phi_{i}$ comes almost for free as $S_{i} \Phi_{i}=-C_{i}^{T} \Lambda_{i}$ (see (22)). Once $S_{i} \Phi_{i}$ is computed, the computation of $\Phi_{i}^{t} S_{i} \Phi_{i}$ just requires a further dense matrixmatrix multiplication, which for portability and efficiency, is performed using the corresponding kernel in the level 3
BLAS. On the other hand, the computation of the contribution of subdomain $i$ to the coarse-grid residual during preconditioner application, i.e., $\Phi_{i}^{t} r_{i}$, is most conveniently carried out with a level 2 BLAS matrix-vector multiplication.

Finally, the coarse-grid preconditioning level is responsible for the assembly, the Cholesky factorization of $S_{0}$ and the solution of the coarse-grid system during preconditioner set-up and application, respectively. For convenience, we split the presentation into these two phases.

Coarse Preconditioner Set-up In a first symbolic phase, the adjacency graph of the sparse coarse-grid coefficient matrix $S_{0}$ is built on one or all processors. Two steps are required: (a) the computation of a global ordering of coarse-grid nodes (objects for BDDC and subdomains for BNN); (b) the computation of the global (although sparse) coupling among coarse-grid nodes. Step (a) is naive in the case of the BNN method, as this ordering already coincides with the global ordering of the subdomains. However, in the case of the BDDC method, its construction requires that one or all processors gather all the coarse-grid nodes each MPI task has identified on its local interface. The labeling of coarse-grid nodes is greatly simplified in our implemen-

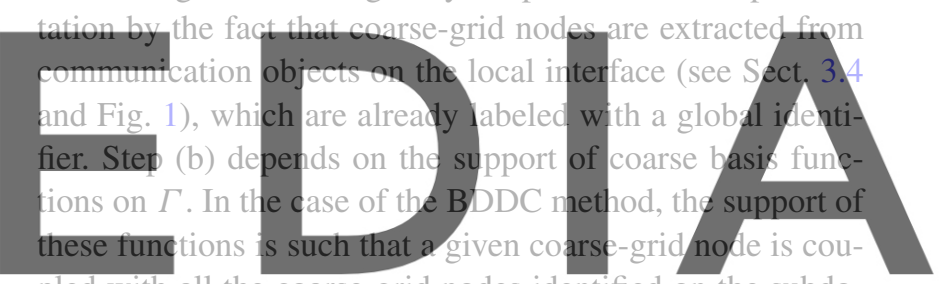
pled with all the coarse-grid nodes identified on the subdomains that surround the node. Therefore, it is sufficient that

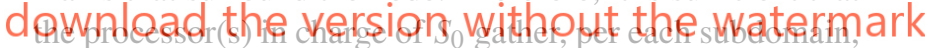

the list of subdomains that surround each coarse-grid node. In case of the BNN method, the support of basis functions is such that two coarse-nodes are coupled if they are neighbors or neighbors of neighbors. Therefore, to determine the sparsity pattern of $S_{0}$, it is sufficient to gather, per each subdomain, its list of neighboring subdomains. This global data structure is referred on the literature as subdomain graph or partition graph (see, e.g. [31, 33, 34]). In a second numerical phase, the matrix $S_{0}$ is both assembled and then factorized on one or all processors. In order to do so, processor(s) in charge of $S_{0}$ gather subdomain local contributions $\Phi_{i}^{t} S_{i} \Phi_{i}$, and then assemble them into $S_{0}$. The correspondence among the entries of each subdomain elemental matrix and $S_{0}$ for this assembly process is given by the global ordering (pre)computed on step (a).

Coarse Preconditioner Application The processor(s) responsible for the coarse-grid problem solution first gather subdomain local contributions (see (15) and then assemble them to build the coarse-grid residual. The backward/forward substitution with the Cholesky factor of $S_{0}$ 


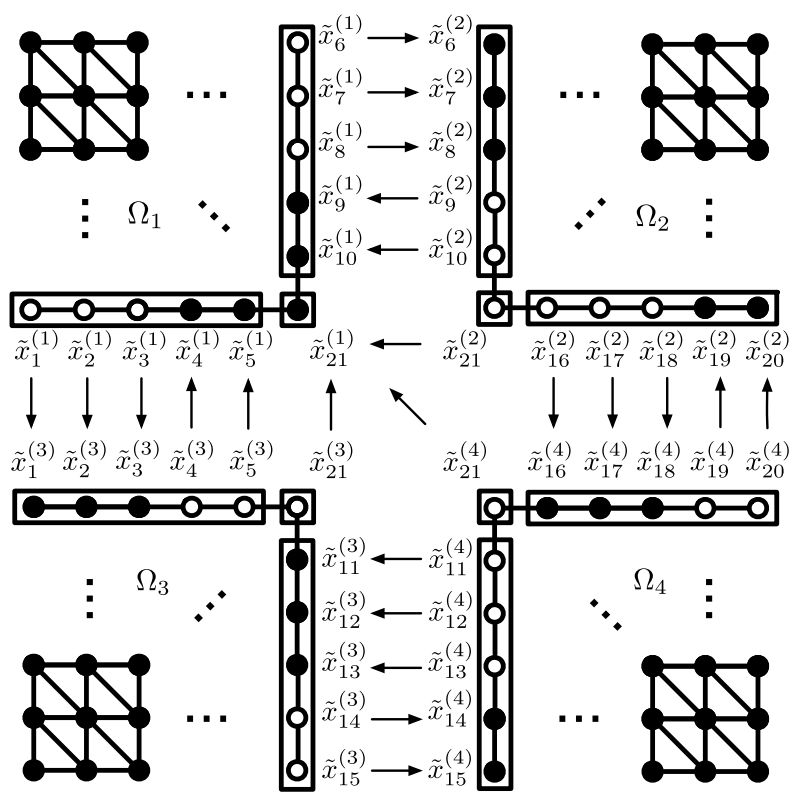

(a)

Fig. 1 Illustration of the two nearest neighbor exchange phases required to obtain a distributed vector stored in fully summed form from the same distributed vector stored in partially summed form. The computational mesh has been partitioned into 4 subdomains, with 5 communication objects on the interface. Four communication objects

provides the preconditioned coarse-grid residual, which is then distributed among all processors only in case one processor is responsible for the solution of the coarse-grid residual.

\subsection{Local Nearest Neighbor Exchange Communications}

A basic communication kernel to be implemented in nonoverlapping DD preconditioners is the one that given a distributed vector stored in partially summed form returns after communication the same distributed vector stored in fully summed form, see (6) and (14). In contrast to global dense collectives such as all-to-all, gather or scatter, this collective communication is highly sparse as it only requires communication among (a moderate number of) nearest neighbors. For example, for structured meshes, each part has only 8 and 26 neighbors in 2D and 3D, respectively. An implementation of this kernel requires a representation of the local interface of each subdomain in terms of communication objects. Communication objects embrace all the nodes of the mesh interface that are shared by the same subdomains. Each mesh point residing on the interface is assigned an owner subdomain. The rest of subdomains sharing this mesh point automatically become non-owners. See Fig. 1 for a graphical representation of these concepts. In a first data exchange among nearest neighbors, non-owner subdomains send their local contributions to the owner subdomain, while the owner

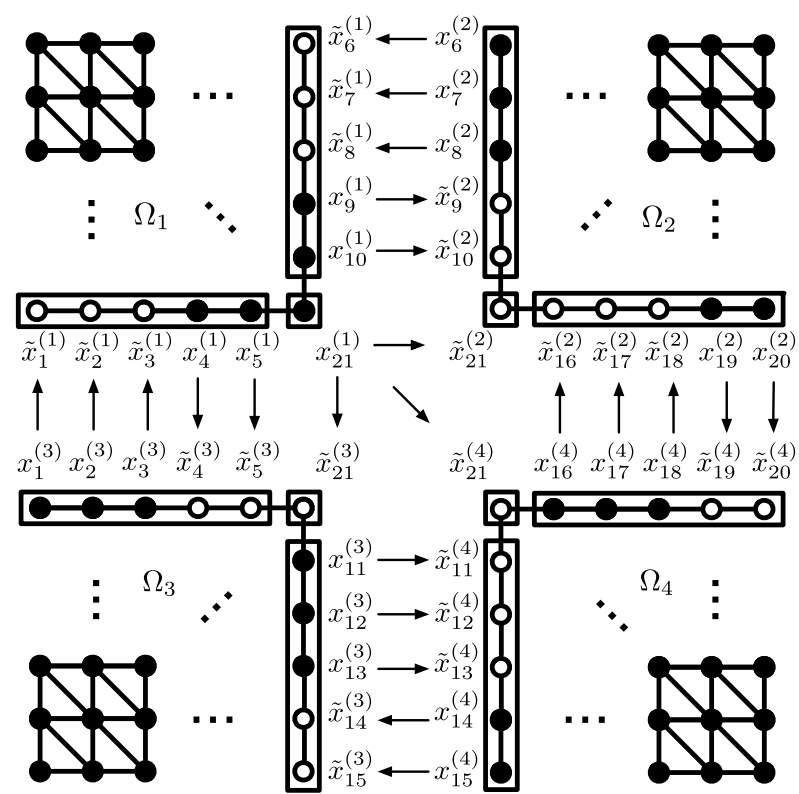

(b)

are shared by subdomains $1-3,1-2,3-4$, and 2-4, respectively, while the remaining object is shared among all subdomains. The owner subdomain of a given interface point is represented in black, while a non-owner one in white

subdomain is in charge for data accumulation and update to obtain fully summed entries. This is illustrated in Fig. 1(a). For example, subdomains 2,3 and 4 send $\tilde{x}_{21}^{(2)}, \tilde{x}_{21}^{(3)}$ and $\tilde{x}_{21}^{(4)}$, respectively, while subdomain 1 receives and accumulates them to obtain $x_{21}^{(1)}$. Then, in a second data exchange, owners send copies of its fully summed entries to the non-owner sides, which just copy them into their local data structures. This exchange phase is illustrated in Fig. 1(b). The strategy based on owner and non-owner subdomains of mesh interface points is also covered in [34].

There are two main implementation issues that significantly influence the performance and scalability of the data exchanges among nearest neighbors. The first one targets the strategy to determine the owner subdomain of each mesh interface point, as this strategy influences the trade-off among message size and number of messages to be exchanged. Our implementation relies on a very simple strategy (that we demonstrate afterwards to be quite efficient): (1) objects shared by two subdomains are divided equally among them (i.e., one subdomain is the owner of the first half, while the other of the second half), so that message sizes to be exchanged on each side are balanced; (2) objects shared by more than two parts are (arbitrarily) owned by the part with minimum identifier. This results in a trade-off which chooses a smaller number of larger messages over a larger number of smaller messages (e.g., assuming a given object is shared among $n$ parts, a $n$-to- 1 followed by a 1 -to- $n$ com- 


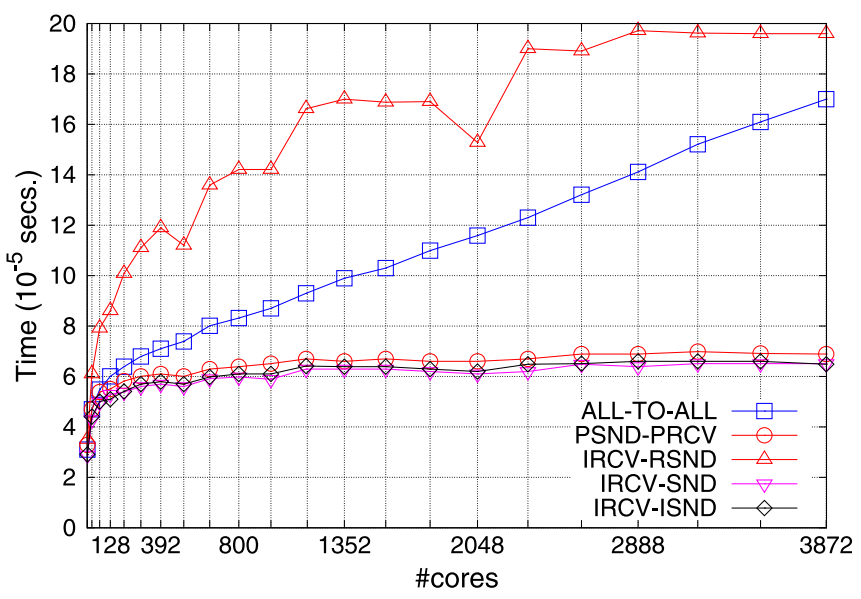

(a)

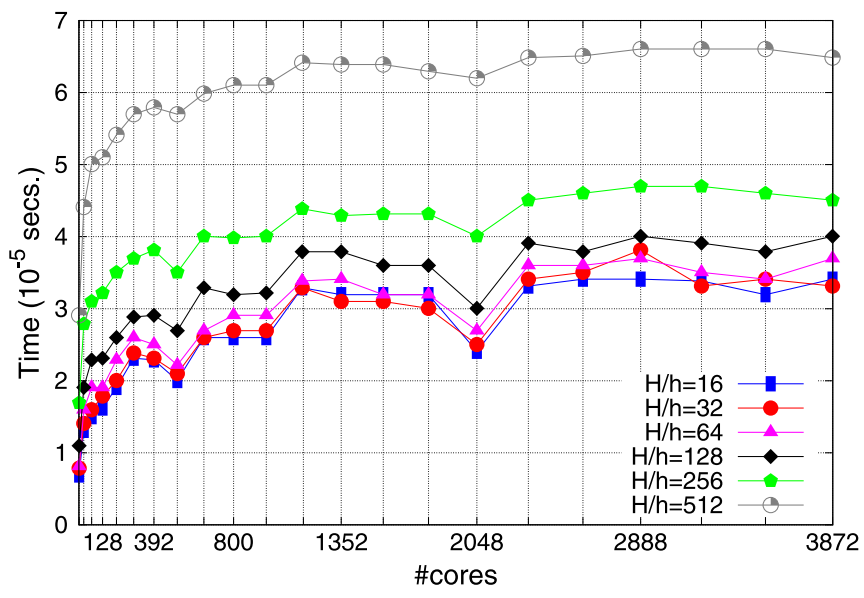

(b)

Fig. 2 Weak scaling for the two exchange phases illustrated in Fig. 1. (a) Several implementations and fixed local problem size $\frac{H}{h}=512$. (b) IRCV_ISND implementation for several local problem sizes $\frac{H}{h}$

munication pattern is preferred over two consecutive $n$-to- $n$ communication patterns with smaller messages). The second issue targets the MPI implementation of each data exchange phase, as the MPI standard does not currently support sparse collectives. There are two possible solutions to bypass this limitation: (a) to use existing MPI dense collectives, in particular, irregular (vector), personalized allto-all exchange, in which no data are exchanged between processes that are not neighbors (i.e., MPI_Alltoallv). This solution has several disadvantages as discussed in detail in [25]; (b) to implement the sparse collective by means of point-to-point communication operations. This is the solution followed by the vast majority of existing distributedmemory linear algebra codes. After a comprehensive literature (see, e.g. [21, 22]) and parallel software (e.g., TRILINOS [23, 24], PETSC [4, 5], PSBLAS [14, 15]) review, existing general-purpose solutions can be categorized as follows depending on the order on which the send/receive operations are issued and the particular blocking semantics of the point-to-point communication operations:

- PSND-PRCV. Each MPI rank traverses its local neighborhood and issues a send operation per neighbor. Then, in a second traversal, it issues a receive operation per neighbor. Send operations are locally blocking while receive operations are blocking (i.e., MPI_Recv). Locally blocking semantics ensure that the send operation immediately returns the control to the application after the message to be sent has been copied in an intermediate buffer and therefore avoids the potential deadlock of blocking sends (i.e., MPI_Send). This is the strategy followed by the PSBLAS library, which in turn inherited the locally blocking semantics from the BLACS [15].

- IRCV-RSND. All receive operations are issued first, then all send operations. Receive operations are non-blocking (i.e., MPI_IRecv), while send operations are ready blocking (i.e., MPI_Rsend). A global barrier operation (i.e., MPI_Barrier) is issued between receive and send operations as required by the ready blocking semantics. At the end, processes wait for the non-blocking receive operations to complete (i.e., MPI_Waitall). This is the strategy implemented in Epetra-Trilinos [23].

- IRCV-SND. Same as IRCV-RSND but ready blocking sends are replaced by blocking sends (i.e., MPI_Recv). No barrier is required in between sends and receives.

- IRCV-ISND. Same as IRCV-SND but blocking sends are replaced by non-blocking sends (i.e., MPI_ISend). At the end, processes wait for both the non-blocking receives and send communication operations.

In order to provide some evidence with respect to the performance and scalability of the communication kernel covered in this section, Fig. 2 illustrates typical weak scaling curves for the parallel execution time required to perform the two nearest neighbor exchange phases on the HPC-FF (see Sect. 4.1). We focus on 2D structured meshes of quadrilateral elements, although similar conclusions can be raised in the 3D structured case. We refer to Sect. 4.1 for a detailed description of the experiment set-up. Figure 2(a) compares the parallel execution time of the aforementioned MPI implementations when increasing the number of cores while keeping fixed the local problem size to $\left(\frac{H}{h}\right)^{2}=512^{2}$ quadrilaterals. This figure shows that PSND-PRCV, IRCV-SND and IRCV-ISND are weakly scalable MPI implementations, as they reach asymptotic parallel execution time (of approximately $65 \mu$-seconds). However, the performance of IRCVRSND significantly degrades with the number of cores. On the HPC-FF, it seems that any performance gain obtained as by-product of the ready blocking semantics (hand-shaking and intermediate buffer copying removal) does not pay off 
the overhead associated to the barrier required in between receives and sends, which introduces a global synchronization point that limits the amount of parallelism by exacerbating the effects of load imbalance. The performance and scalability of the implementation based on MPI_Alltoallv is not surprising as it does not properly capture the parallelism inherent to the sparse communication pattern (as pointed out in [25]). Figure 2(b) illustrates the results of the same weak scaling study of Fig. 2(a), but it focus on one of the three most efficient implementations (i.e., IRCV-ISND) with problem size fixed to several values. Absolute timings and the order of complexity with $\frac{H}{h}$ shown in Fig. 2(b) reveal that, as long as a weak scalable implementation such as IRCV-ISND is employed, the contribution of this communication kernel to the overall performance of BDD methods can be considered negligible compared to that of other building blocks in this family of algorithms, such as, e.g., the solution of local Dirichlet problems or the computation of a coarse-grid correction.

\subsection{Global Collectives}

The global MPI communication operations required for the implementation of the coarse-grid preconditioning level in the one processor case are MPI_Gatherv (twice for the preconditioner set-up and once per preconditioner application) and MPI_Scatterv (once per preconditioner application), while MPI_Allgatherv (twice for the preconditioner set-up and once per preconditioner application) is required in case the coarse-grid problem is solved on all processors. These varying message size collectives are the ones that most accurately capture the global communication pattern involved in the (serial) solution of the coarse-grid problem. For structured meshes and regular partitions, boundary subdomains do not send/receive the same amount of data than internal ones, and for unstructured ones, the shape of each subdomain local interface is strongly dependent on the underlying (irregular) non-overlapping partition. However, we have experimentally observed on several distributed-memory platforms that the performance of the solution of the coarse-grid problem may benefit from exploiting fixed message size collectives, namely MPI_Gather/MPI_Scatter/MPI_Allgather. Figure 3 depicts what we have observed on the HPC-FF supercomputer. In particular, it reports the parallel execution time with increasing number of cores for fixed and varying message size collectives for powers-of-two message sizes in the range 32-8192 bytes; all message sizes exchanged by BDD methods in the case of structured 2D/3D meshes are enclosed within this range. Figure 3 reveals that for message sizes below or equal to 512 bytes the performance of MPI_Gather is superior to that of MPI_Gatherv, and the smaller the message size the more superior MPI_Gather over MPI_Gatherv. We can observe just the opposite for message sizes beyond 512 bytes, with the largest gains of MPI_Gatherv over MPI_Gather with the largest message sizes. In the case of scatter communication, a much larger message size of approximately 4096 bytes is required by MPI_Scatterv to become superior to MPI_Scatter. Besides, for "small" message sizes, larger gains of MPI_Scatter over MPI_Scatterv are attained compared to those observed for gather communication. Finally, the performance of MPI_AllGatherv and MPI_AllGather collectives is almost coincident.

\section{Scalability Study}

\subsection{Experimental Framework}

The algorithms subject of study were implemented in FEMPAR, an in-house, developed from scratch, OO framework which, among other features, provides the basic tools for the efficient message-passing (MPI) implementation of substructuring DD solvers, using METIS [26] for unstructured meshes. All experiments reported in the sequel were obtained on a large-scale multicore-based distributed-memory machine, the HPC-FF (HPC for Fusion), located at the Juelich (Germany) Supercomputing Centre. The HPC-FF is a QDR Infiniband interconnected commodity cluster composed of 1080 Bull NovaScale R422-E2 blades. Each blade is equipped with two Intel Xeon X5570 QuadCore processors running at $2.93 \mathrm{GHz}$ (8 computational cores in total) and 24 GBytes of DDR3 memory, and runs a fullfeatured SUSE SLES 11 Linux OS. The codes were compiled using Intel Fortran compiler (12.1.4) with recommended optimization flags and we used Parastation 5.0 MPI tools and libraries for native message-passing. The codes were linked against the BLAS/LAPACK and PARDISO available on the Intel MKL library (version 10.3, build 10). Peak flop performance per core is 11.72 GFLOPs/sec (i.e., $93.76 \mathrm{GFLOPs} / \mathrm{sec}$ per blade) and measured MPI intrasocket, intersocket and internode latency and bandwidth for this machine are $0.26 \mu$-seconds and 4.6 GBytes/sec, 0.57 and 3.7, and 1.49 and 3.1, respectively. We stress that we have also evaluated the codes on several radically different platforms (e.g., MareNostrum, a Myrinet-interconnected cluster composed of 2560 IBM JS21 compute nodes at the Barcelona Supercomputing Center). We skip the corresponding results because similar balances to those reported next for the HPC-FF were achieved.

The experimental study in this paper focuses on the evaluation of the weak scalability of several sub-structuring DD solvers when applied to the Poisson problem. Recall that weak scaling studies determine at which rate a given magnitude evolves with the number of cores $P$ while keeping the local problem size $\frac{H}{h}$ constant. In particular, mag- 


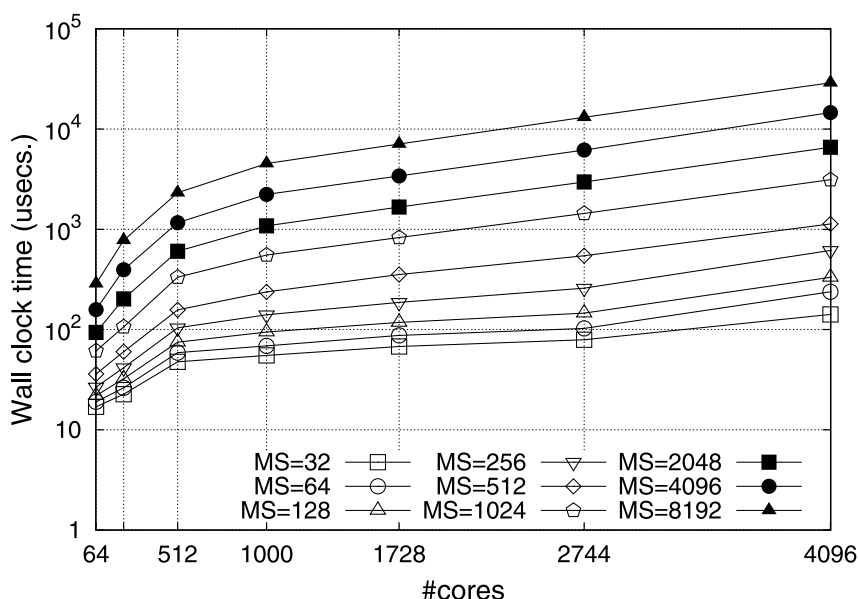

(a)

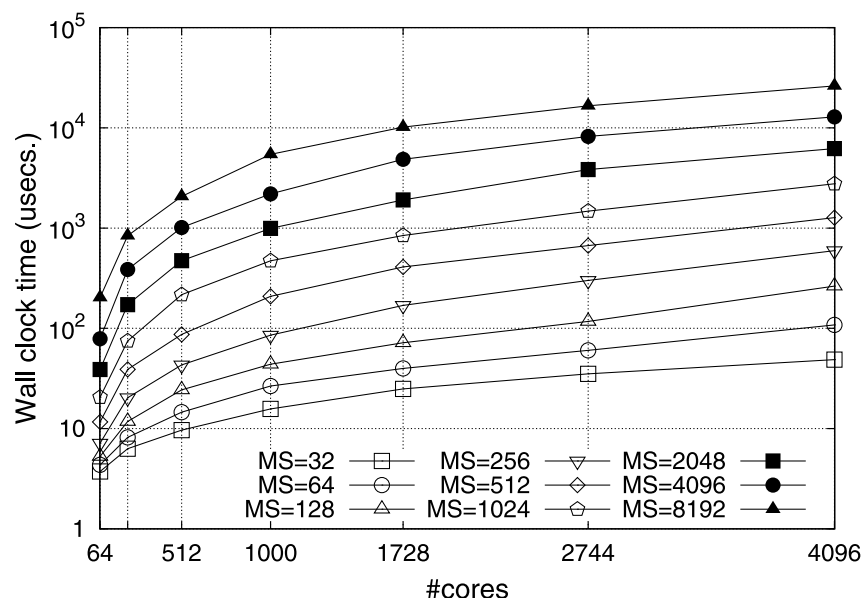

(c)

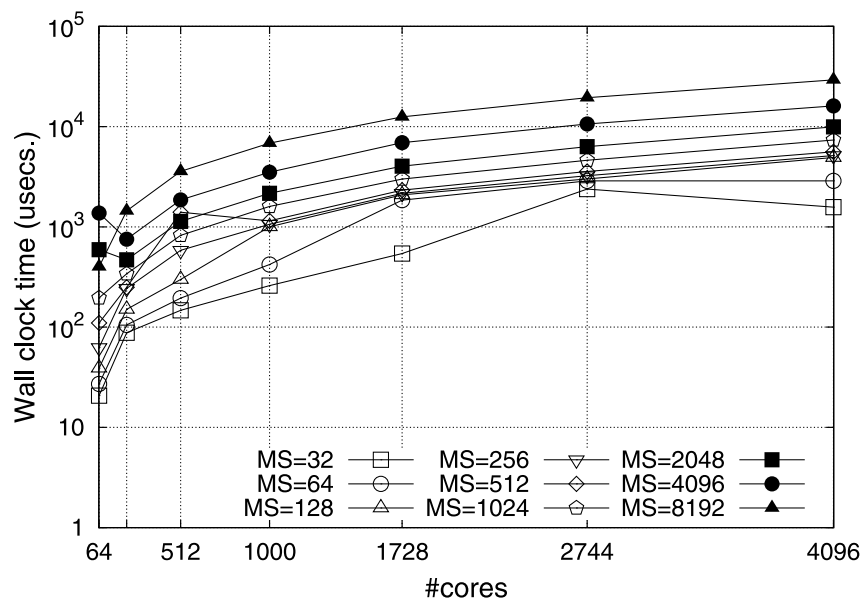

(e)

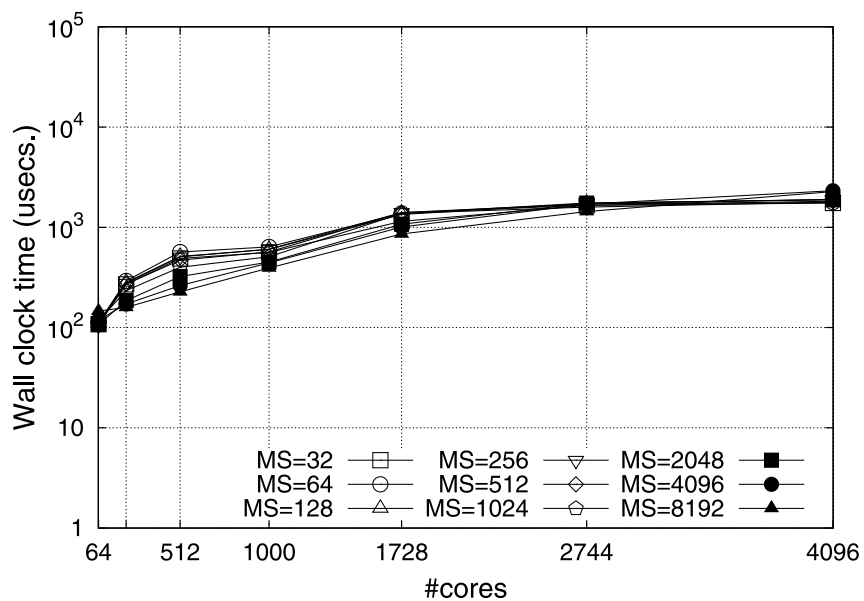

(b)

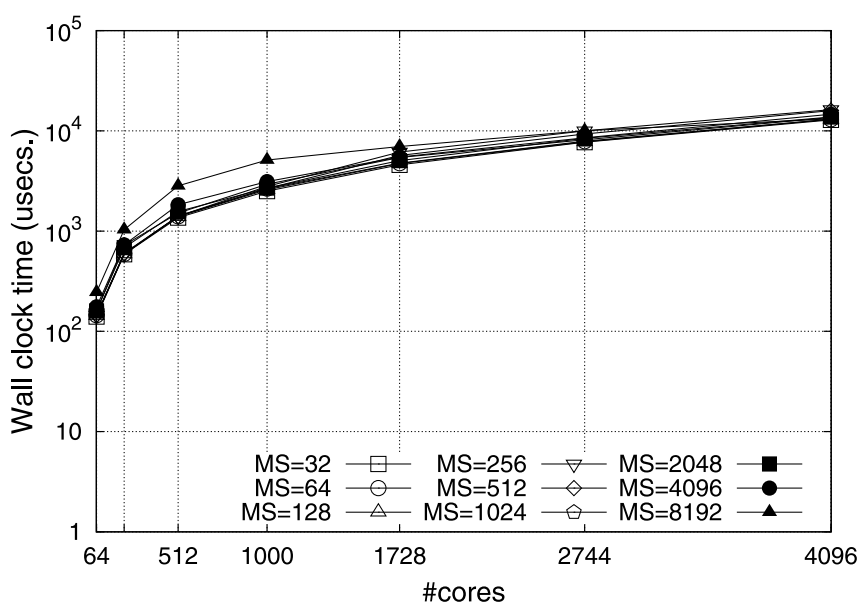

(d)

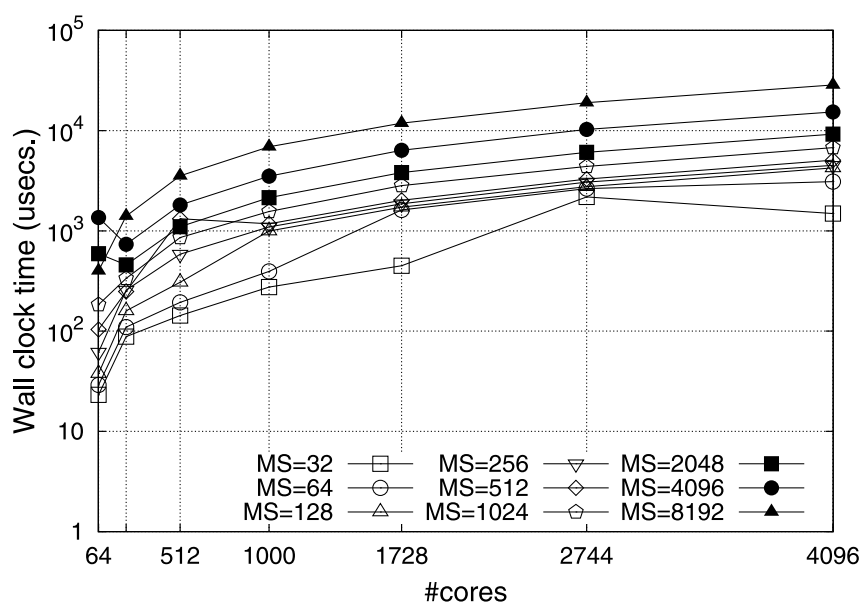

(f)

Fig. 3 Performance and scalability of fixed and varying message size collectives on the HPC-FF supercomputer. Fixed: (a) MPI_Gather; (c) MPI_Scatter; (e) MPI_Allgather. Varying: (b) MPI_Gatherv; (d) MPI_Scatterv; (f) MPI_Allgatherv

nitudes of interest for our study are the total computation time, and the number of PCG iterations required to solve the preconditioned interface problem (3). The former magnitude is in turn concentrated on three phases: the Schur- complement system and preconditioner set-up, and the iterative solution of (3) by the PCG Krylov subspace solver. In Sects. 4.1.1, and 4.1.2 we introduce the particular ranges of $P$ and $\frac{H}{h}$ that our study explores for $2 \mathrm{D}$ and $3 \mathrm{D}$, respec- 
Table 1 Arithmetic complexities of the different stages in the serial direct solution of sparse linear systems

\begin{tabular}{lll}
\hline Phase & 2D complexity $(d=2)$ & 3D complexity $(d=3)$ \\
\hline Reordering & $\mathcal{O}(n)$ & $\mathcal{O}(n)$ \\
Symbolic factorization & $\mathcal{O}(n \log n)$ & $\mathcal{O}\left(n^{\frac{4}{3}}\right)$ \\
Numerical factorization & $\mathcal{O}\left(n^{\frac{3}{2}}\right)$ & $\mathcal{O}\left(n^{2}\right)$ \\
Triangular solution & $\mathcal{O}(n \log n)$ & $\mathcal{O}\left(n^{\frac{4}{3}}\right)$ \\
\hline
\end{tabular}

Table 2 Parallel complexity for the NN-PCG and BDD-PCG methods. Sparse direct solvers are assumed as well as the serial solution of the coarse-grid problem. It is obtained as the sum of the set-up phase complexity, with unknown constant $c_{s}$, and the iterative phase com- plexity, with unknown constant $c_{i}$. In the case of BDD-PCG methods, the unknown constants $c_{s}$ and $c_{i}$ are separated into coarse-grid (c) and fine-grid (f) preconditioning contributions

\begin{tabular}{lll}
\hline Method & 2D complexity $(d=2)$ & 3D complexity $(d=3)$ \\
\hline NN-PCG & $c_{s} n^{\frac{3}{2}}+c_{i} \sqrt{P} n \log (n)$ & $c_{s} n^{2}+c_{i} \sqrt[3]{P} n^{\frac{4}{3}}$ \\
BDD-PCG & $c_{s f} n^{\frac{3}{2}}+c_{s c} P^{\frac{3}{2}}+c_{i f} n \log (n)+c_{i c} P \log P$ & $c_{s f} n^{2}+c_{s c} P^{2}+c_{i f} n^{\frac{4}{3}}+c_{i c} P^{\frac{4}{3}}$ \\
\hline
\end{tabular}

tively, and how the problem is mapped to the underlying computer.

\subsubsection{Set-up for 2D Experiments}

We consider the solution of the Poisson problem on a rectangle $\bar{\Omega}=[0,2] \times[0,1]$, a global conforming uniform mesh (partition) of $\bar{\Omega}$ into quadrilaterals, and a bilinear finite element discretization (i.e., Q1-elements). The 2D mesh was partitioned into rectangular grids of $P=4 m \times 2 m$ square subdomains, and distributed over $m=1,2, \ldots, 22$ nodes, with $4 \times 2$ subdomains/MPI Ranks per node and one MPI Rank per core of the HPC-FF. In order to evaluate the weak scaling of the solvers under several computation/communication balances, we consider increasing values for $\frac{H}{h}=16,32,64,128,256$ and 512, with the two extremes being the most and least communication-bounded scenarios of the sample. The largest problem size $\frac{H}{h}=512$ was selected strategically to be the largest power-of-two that fits into the machine given a memory limit per core of 1.7 GBytes. Note that in 2D the number of quadrilaterals on each local mesh is therefore $\frac{H}{h} \times \frac{H}{h}$, and that of the global mesh is given by $4 m \frac{H}{h} \times 2 m \frac{H}{h}$.

\subsubsection{Set-up for 3D Experiments}

We consider the solution of the Poisson problem on a cube $\bar{\Omega}=[0,1] \times[0,1] \times[0,1]$, a global conforming uniform mesh (partition) of $\bar{\Omega}$ into hexahedra and a trilinear finite element discretization (i.e., Q1-elements). The 3D mesh is partitioned into cubic grids of $P=2 m \times 2 m \times 2 m$ cubic subdomains and distributed over $m=1,2, \ldots, 8$ nodes, with $2 \times 2 \times 2$ subdomains/MPI Ranks per node and one MPI Rank per core of the HPC-FF. We consider increasing values for $\frac{H}{h}=10,20,30$ and 40. The largest problem size $\frac{H}{h}=40$ was selected strategically to be the largest multiple-of-ten that fits into the machine given a memory limit per core of 1.7 GBytes. Note that in 3D the number of hexahedra on each local mesh is $\frac{H}{h} \times \frac{H}{h} \times \frac{H}{h}$, and that of the global mesh is given by $2 m \frac{H}{h} \times 2 m \frac{H}{h} \times 2 m \frac{H}{h}$.

\subsection{A Simple Computational Model}

Table 1 summarizes the well-known [16] order of arithmetic complexity of the different stages of the serial direct solution of sparse linear systems arising from the discretization of a square or cube with a uniform mesh with $n$ nodes, with $d=2,3$ the dimension of the space.

If the uniform mesh is distributed over a uniform subdomain grid with $P=H^{-d}$ subdomains, with $n=\left(\frac{H}{h}\right)^{d}$ nodes on each subdomain, the estimated complexity the NN-PCG and BDD-PCG methods is given on Table 2. Recall that, in our codes, Reordering, Symbolic Factorization and $\mathrm{Nu}-$ merical Factorization are performed during preconditioner and Schur complement set-up, while Triangular Solution is performed at each PCG iteration during Schur complement and preconditioner application. The complexity of reordering and symbolic factorization has been omitted in the table. Besides, for simplicity, the effect of communication and load unbalancing has been neglected. Constants $c_{S}$ and $c_{i}$ depend on the particular stencil the sparse direct method is applied to (linear FEs, quadratic FEs, etc.), and also on the ability of the software for the efficient exploitation of the underlying machine characteristics. For BDD-PCG methods, $c_{i}$ actually depends on $n$, although this dependence is very mild (see e.g. (9)) and can be neglected as stated in Sects. 2.3 and 2.4 .

The simple computational model in Table 2 reveals that the scalability of NN-PCG and BDD-PCG is composed of two components, a scalable one that does not depend on $P$, 


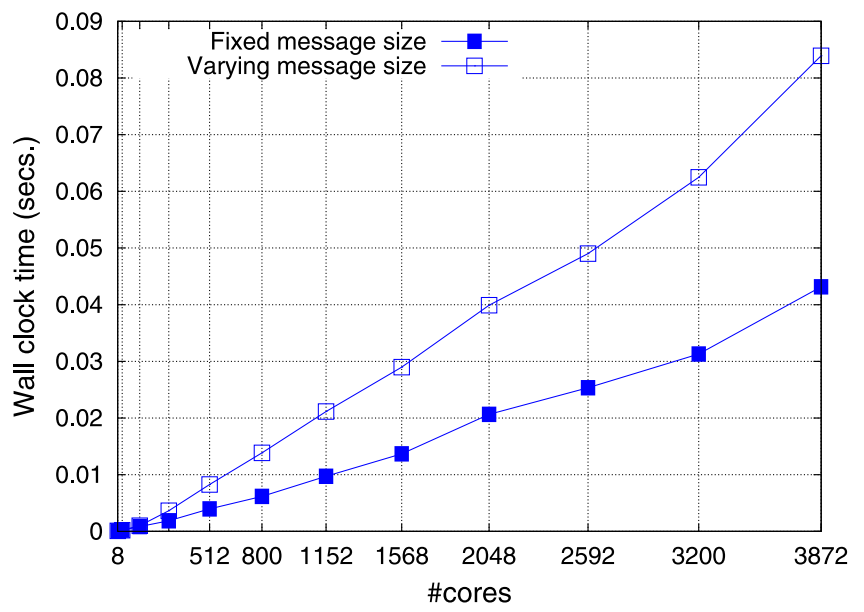

(a)

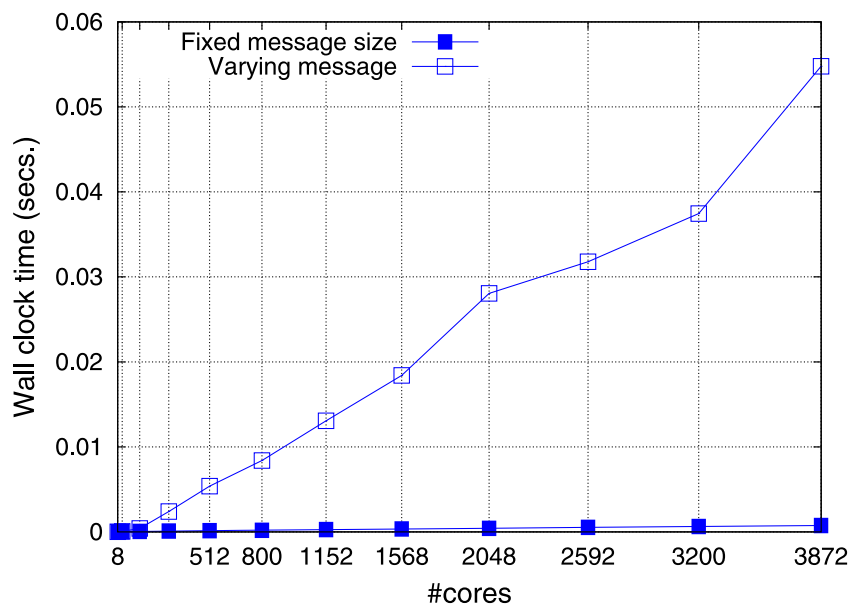

(c)

Fig. 4 Weak scalability for the parallel execution time of the coarsegrid preconditioner set-up ((a) and (b)) and application $((\mathbf{c})$ and $(\mathbf{d}))$ for the 2D Poisson problem on the HPC-FF. (a) and (c): BDDC(c) preconditioner, one processor is responsible for coarse-grid problem duties,

and a non-scalable one that grows with $P$. In the former method, the non-scalable component is associated with the lack of a coarse-grid correction and subsequent degradation of PCG convergence rates with $P$ (see Sect. 2.2). This is inherent to the preconditioning approach and cannot be mitigated. In the latter method, the non-scalable component is associated to the extra cost of the solution of the coarsegrid problem, and there is a lot of margin for improvement of this term via high-performance computing techniques (e.g., fine-grid/coarse-grid overlapping, distributed-memory implementation of coarse-grid preconditioning level). Anyway, the key of BDD-PCG methods is that the non-scalable component does not depend on $\frac{H}{h}$. This means that, as long as $\frac{H}{h}$ is "large enough", a balance among the non-scalable and scalable components can be reached such that the latter determines the overall (weak) scalability of the solution. The purpose of Sects. 4.3-4.4 is to demonstrate that, with

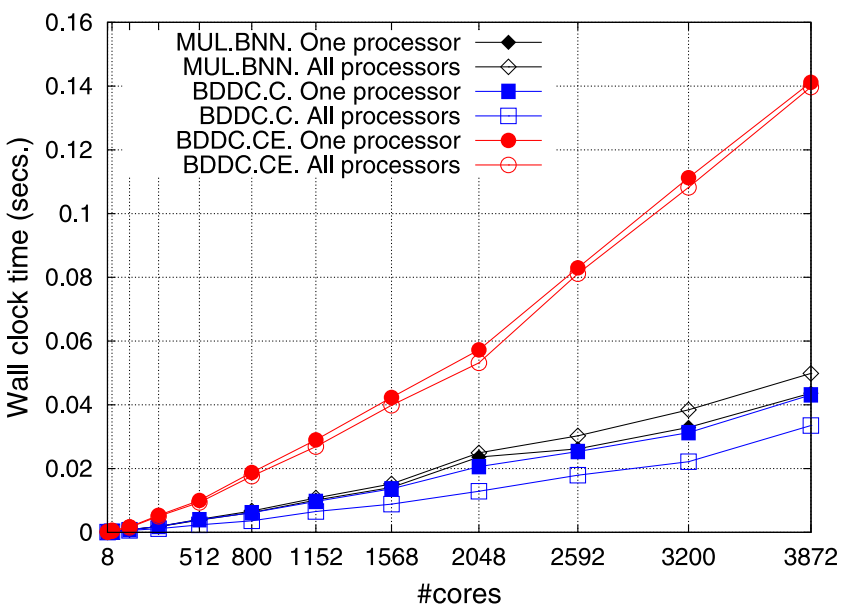

(b)

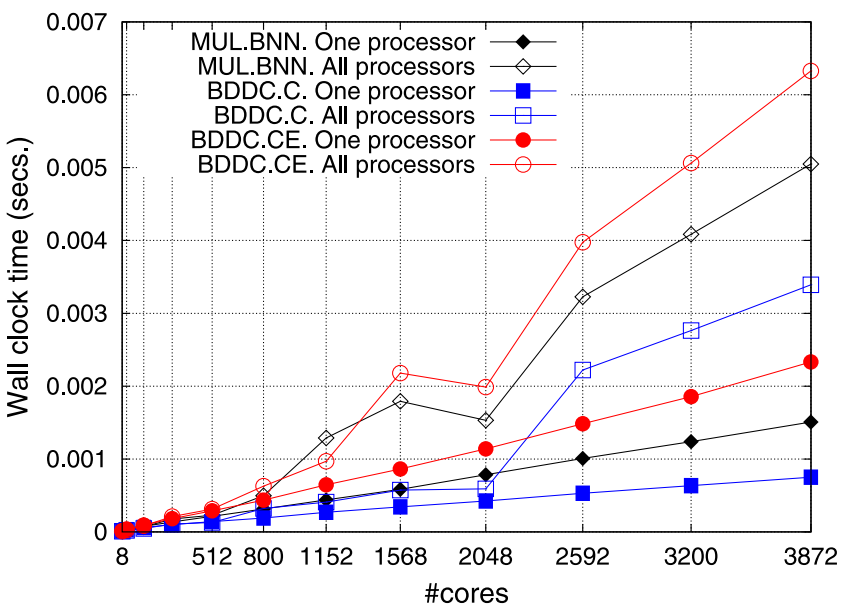

(d)

comparison of fixed and varying message size collectives. (b) and (d): comparison of $\mathrm{BDDC}(\mathrm{c}), \mathrm{BDDC}(\mathrm{ce})$ and $\mathrm{BNN}$ preconditioners with one or all processors responsible for coarse-grid problem duties, fixed message size collectives

the current software and distributed-memory machines, constants in Table 2 are such that our implementation can be very efficient for interesting ranges of applicability.

\subsection{Coarse-Grid Preconditioning Weak Scalability}

We first evaluate the weak scalability of the coarse-grid preconditioning level for the BNN and BDDC solvers. Besides, given the scenario depicted in Sect. 3.5, we will determine which is the fastest solution among the following three approaches: solution on one processor with fixed or varying message size collectives, and solution on all processors. Sections 4.3.1 and 4.3.2 cover the 2D and 3D Poisson problems, respectively.

\subsubsection{D Experiments}

Figures 4(a) and (b) illustrate the weak scalability for the parallel execution time of the coarse-grid preconditioner set- 
up and Figs. 4(c) and (d) that of the coarse-grid preconditioner application for the BNN, BDDC(c), and BDDC(ce) solvers. The coarse-grid preconditioner set-up execution times includes both a symbolic phase, where the graph of $S_{0}$ is built and then symbolically factorized, and a numerical phase, where $S_{0}$ is assembled and then factorized by the sparse Cholesky factorization included in PARDISO. Highly parallel computations in this preconditioning level (such as the computation of $\Phi_{k}^{t} S_{k} \Phi_{k}$ or $\Phi_{k}^{t} r_{k}$ ) are excluded from the figure, i.e., only those terms that grow with $P$ in Table 2, have been considered.

Figure 4 reveals that the weak scalability of the coarsegrid preconditioning level degrades as higher core counts are employed. This is caused by the combined effect of the global collectives scalability for increasing number of cores (see Fig. 3) and the serial preconditioner set-up and application. The latter factor results in idling or wasted computation parallel overheads, as the processors just waste their time waiting on a collective (if they do not have coarsesolver duties) or performing replicated computation, respectively. The coarse-grid preconditioning level is therefore a critical component in our current MPI implementation and any improvement can have significant impact on the performance and scalability of the overall solution (which will be later evaluated in Sect. 4.3.2). Figures 4(a) and (c) show one such improvement for the BDDC(c) preconditioner setup and application, respectively, which comes from the use of fixed message size collectives in case one processor is responsible for coarse-grid level duties. This improvement can be justified by looking carefully at message sizes sent/received in the collectives. This in turn strongly depends on the particular algorithm and phase. For the assembling of the coarse-grid residual, each subdomain sends a message size proportional to the number of neighboring subdomains plus one in case of the BNN preconditioner, i.e., 9 elements $\times 8$ bytes/element $=72$ bytes, and to the number of local coarse-grid nodes in case of the BDDC preconditioner, i.e., $4 \times 8=32$ bytes and $8 \times 8=64$ bytes, for the BDDC(c) and BDDC(ce), respectively. These quantities are squared for the (numerical) assembling of $S_{0}$, i.e., $9^{2} \times 8=648,4^{2} \times 8=128$, and $8^{2} \times 8=512$ bytes. As pointed out by the above discussion of Fig. 3, these message sizes are within the ranges where the use of fixed message size collectives can be beneficial over varying message size ones. Another significant improvement can be observed in Fig. 4(d) if one processor instead of all processors is responsible for coarse-grid preconditioning level duties. This can be justified by the superiority of the MPI_ Gather + MPI_Scatter solution over the MPI_Allgather one for "small" message sizes (see Figs. 3(a), (b) and (c)). Indeed, "All processor" curves in Fig. 4(d) reflect the peaks that are observed for MPI_Allgather in Fig. 3(c). Focusing on the winner implementation for each phase and algorithm in Figs. 4(b) and (d), it can be observed that BDDC(ce) is the method with the most expensive coarse-grid preconditioner set-up and application and besides its computational time degrades with the number of cores at the highest rate, followed by the BNN and BDDC(c) solvers. This ranking is not surprising if one takes a closer look at the stencil of the coarse-grid coefficient matrix of each method for structured partitions. BDDC(c) presents the stencil corresponding to the Q1 FE discretization, BNN a more intricate one where neighbors of neighbors in the Q1 FE discretization are also connected, and finally that of BDDC(ce) resembles that of the Q2 FE discretization (after static condensation of interior nodes). The complexity of the sparse direct Cholesky method applied to a uniform grid with $n=P$ grid points is given in Table 1, with the particular stencil only affecting to the constant. Therefore, it is reasonable that the more intricate the stencil the higher the constant, confirming what is observed in Figs. 4(b) and (d).

\subsubsection{D Experiments}

Figures 5(a) and (b) illustrate the weak scalability for the parallel execution time of the coarse-grid preconditioner setup and Figs. 5(c) and (d) that of the coarse-grid preconditioner application for the BNN, BDDC(ce), and BDDC(cef) solvers.

The solution based on fixed message size collectives is superior to the one based on varying message size ones (see Figs. 5(a) and (c)) and the one processor dedicated to coarsegrid problem duties solution is also superior to the all processors one (see Figs. 5(b) and (d)); the justification of these results follows the one for the 2D structured case (although with larger message sizes in 3D). A much more interesting observation is the relative ranking of the BNN, BDDC(ce) and BDDC(cef) coarse-grid preconditioners and the rate at which their weak scalability degrades with the number of cores. As illustrated by Figs. 5(b) and (d), BNN turns to be the method with the cheapest coarse-grid preconditioner setup and application and the one with the smallest rate, followed by the BDDC(ce) and BDDC(cef) in this strict order. Table 3 provides several metrics of the coarse-grid problem that helps to understand this observation, namely the size and number of non-zeros in its sparse coefficient matrix, and the size of the optimal root separator of its adjacency graph. Although the BNN coarse-grid sparse coefficient is denser, it is 4 and 7 times smaller than that of the BDDC(ce) and BDDC(cef), respectively, and its optimal root separator is 1.5 and 2 times smaller than that of the BDDC(ce) and BDDC(cef), respectively. The size of the optimal root separator, which can be used as lower bound for the complexity of the sparse direct Cholesky (actually its cube and square for the factorization and forward/backward substitution, respectively), accurately describes what is observed in Figs. 5(b) and (d). 


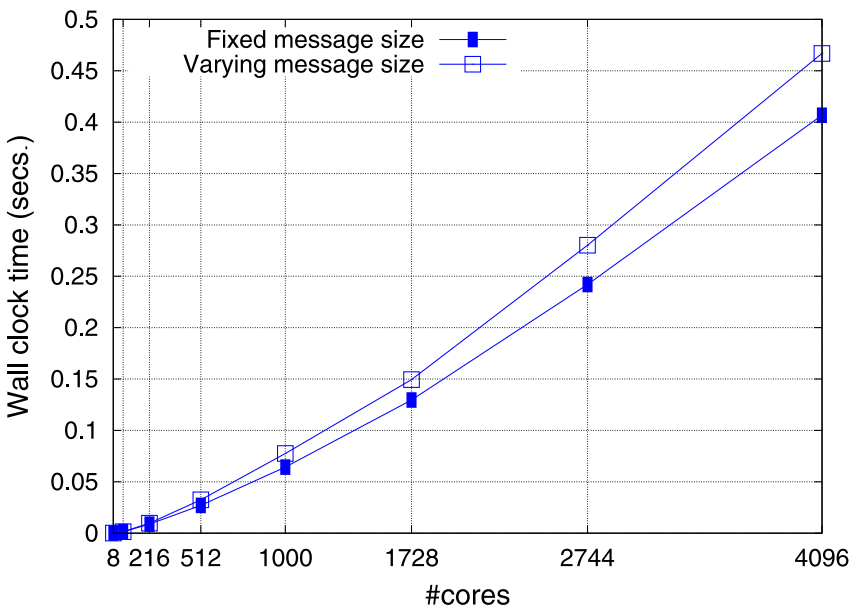

(a)

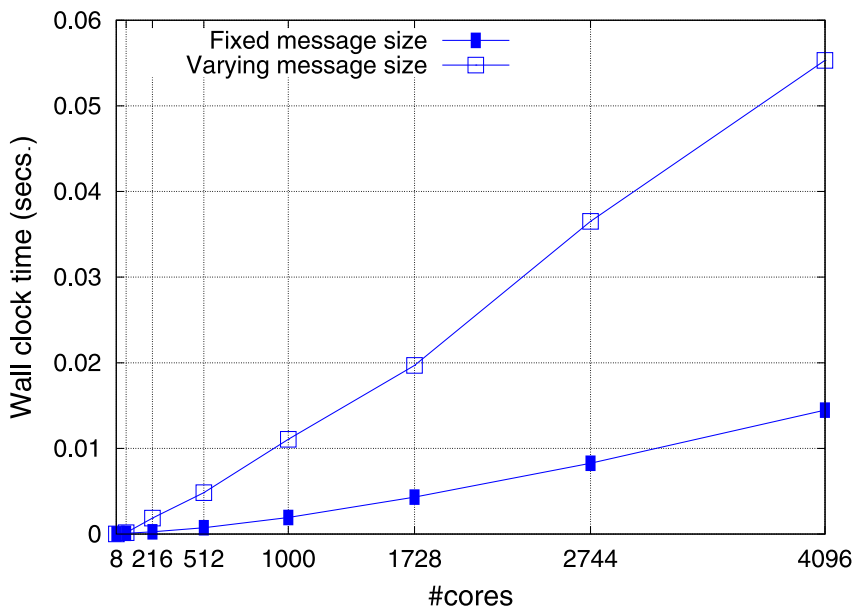

(c)

Fig. 5 Weak scalability for the parallel execution time of the coarsegrid preconditioner set-up ((a) and (b)) and application $((\mathbf{c})$ and $(\mathbf{d}))$ for the 3D Poisson problem on the HPC-FF. (a) and (c): BDDC(ce) preconditioner, one processor is responsible for coarse-grid problem du-

Table 3 Size $\left(n_{c}\right)$, non-zeros $\left(n_{z}\right)$ and optimal root separator size $\left(n_{s}\right)$ for the coarse-grid coefficient matrix in the BDDC and BNN algorithms. A periodic structured mesh of a cube with $P=p^{3}$ subdomains is assumed, with $p$ the number of subdomains per Cartesian direction

\begin{tabular}{llll}
\hline Metric & BDDC (ce) & BDDC (cef) & BNN \\
\hline$n_{c}$ & $4 P$ & $7 P$ & $P$ \\
$n_{z}$ & $234 P$ & $462 P$ & $125 P$ \\
$n_{s}$ & $3 P^{2 / 3}$ & $4 P^{2 / 3}$ & $2 P^{2 / 3}$ \\
\hline
\end{tabular}

\subsection{Overall Scalability}

In this section we take into consideration the overall scalability of the BNN and BDDC solvers. Both fine-grid and coarse-grid preconditioning contributions to the scaling curves are considered, as well as the number of PCG iter-

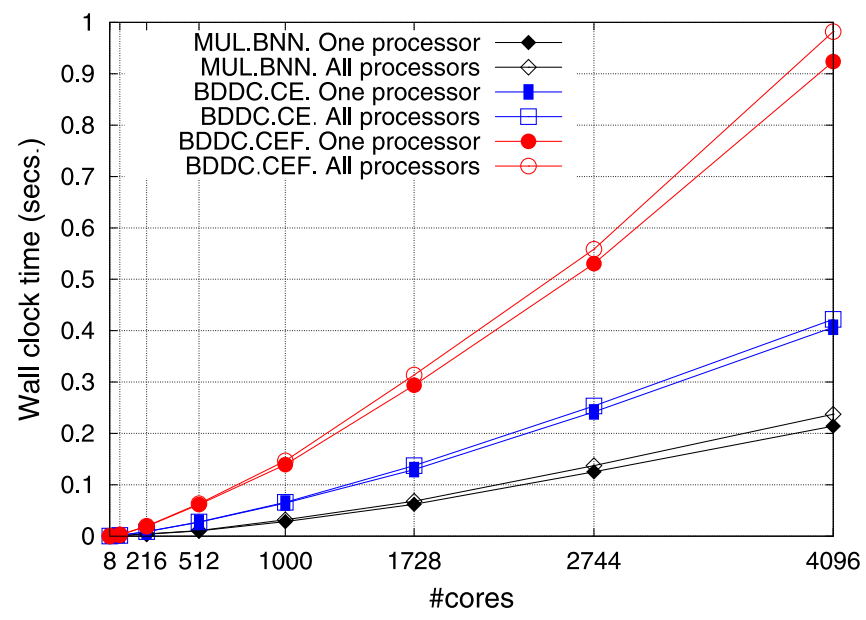

(b)

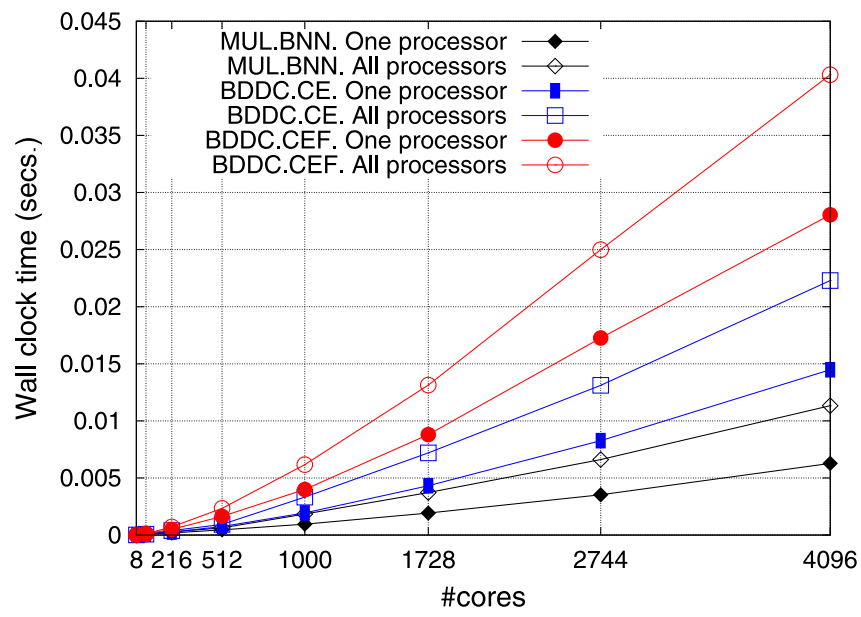

(d)

ties, comparison of fixed and varying message size collectives. (b) and (d): comparison of BDDC(ce), BDDC(cef) and BNN preconditioners with one or all processors responsible for coarse-grid problem duties, fixed message size collectives

ations required to converge. Sections 4.4.1 and 4.4.2 cover the 2D and 3D Poisson problems, respectively.

\subsubsection{D Experiments}

Figure 6 reports the weak scalability for the total computation time of the winner implementation of the multiplicative BNN solver and two different implementations of the BDDC(c) and BDDC(ce) solvers. The best implementation of the coarse-grid preconditioning level was used (see Sect. 4.3). In the legend of the figure, DEF (symmetric-PD) and IND (symmetric INDefinite) refer to the kind of linear systems/solvers that are solved/applied for the computation of the BDDC fine-grid correction (see Sect. 3.2.2). The winner implementation of the BNN method exploits symmetricPD solvers and saves the solution of a Dirichlet solver per 

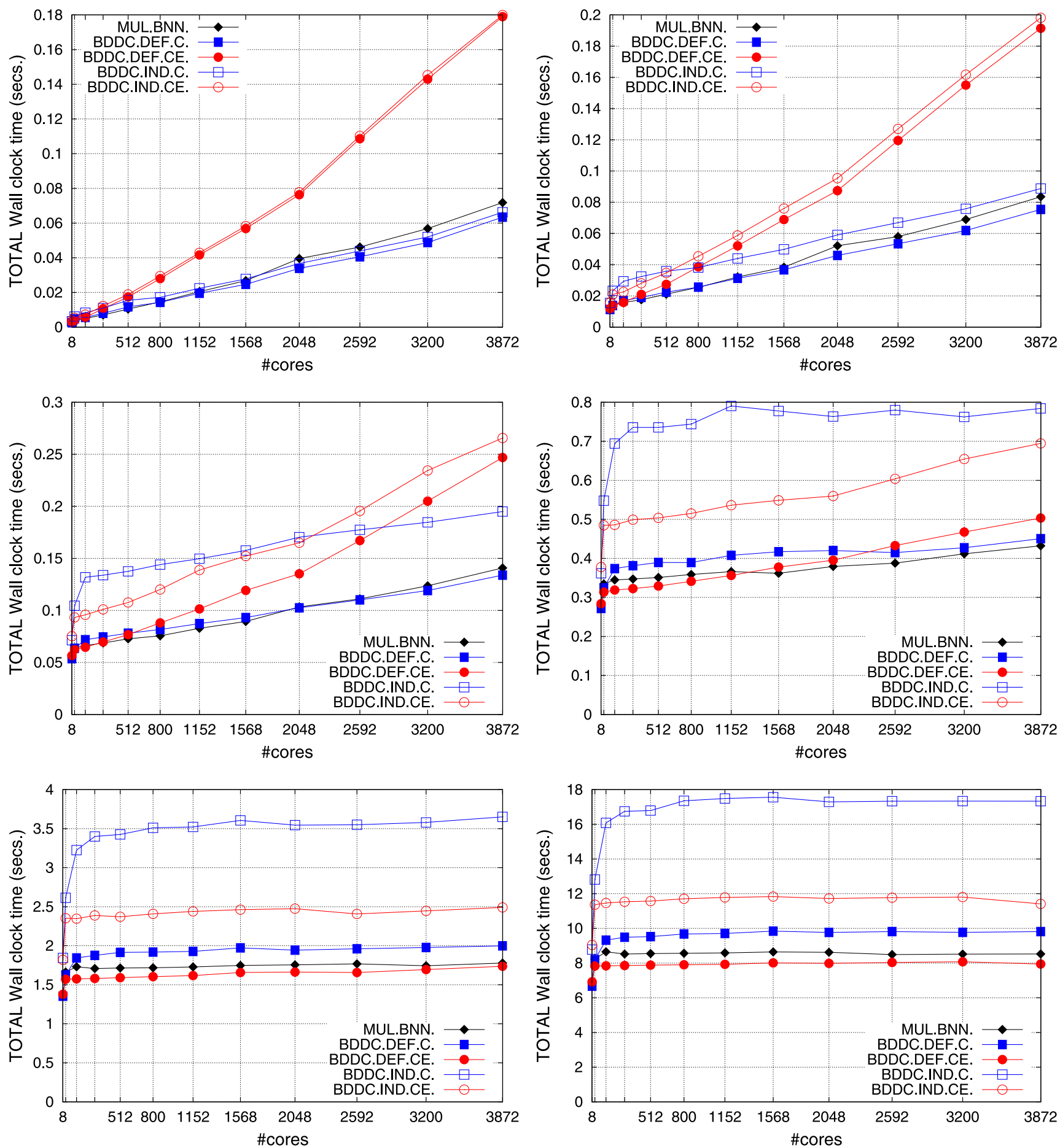

Fig. 6 Weak scalability for the total computation time of the multiplicative BNN (winner implementation) and two different implementations of the BDDC(c) and BDDC(ce) solvers for the 2D Poisson problem on HPC-FF. From top to bottom and left to right $\frac{H}{h}=16,32,64,128,256,512$

PCG iteration (cf. [2]). Figure 7 illustrates the weak scalability for the number of PCG iterations. In the PCG method, we set the initial solution vector guess $x_{0}=0$, and the iteration is stopped whenever the residual $r_{k}$ at a given iteration $k$ satisfies $\left\|r_{k}\right\|_{2} \leq 10^{-6}\left\|r_{0}\right\|_{2}$; this set-up also applies to Sect. 4.4.2.
As predicted by our simple computational model in Sect. 4.2, Fig. 6 clearly evidences that weak scaling curves for the computational time of BNN/BDDC solvers result from the sum of a non-scalable and a scalable component. For "sufficiently small" $\frac{H}{h}$ (e.g., with $\left.\frac{H}{h}=16,32,64\right)$, the non-scalable component (i.e., the one that grows with $P$ ) 

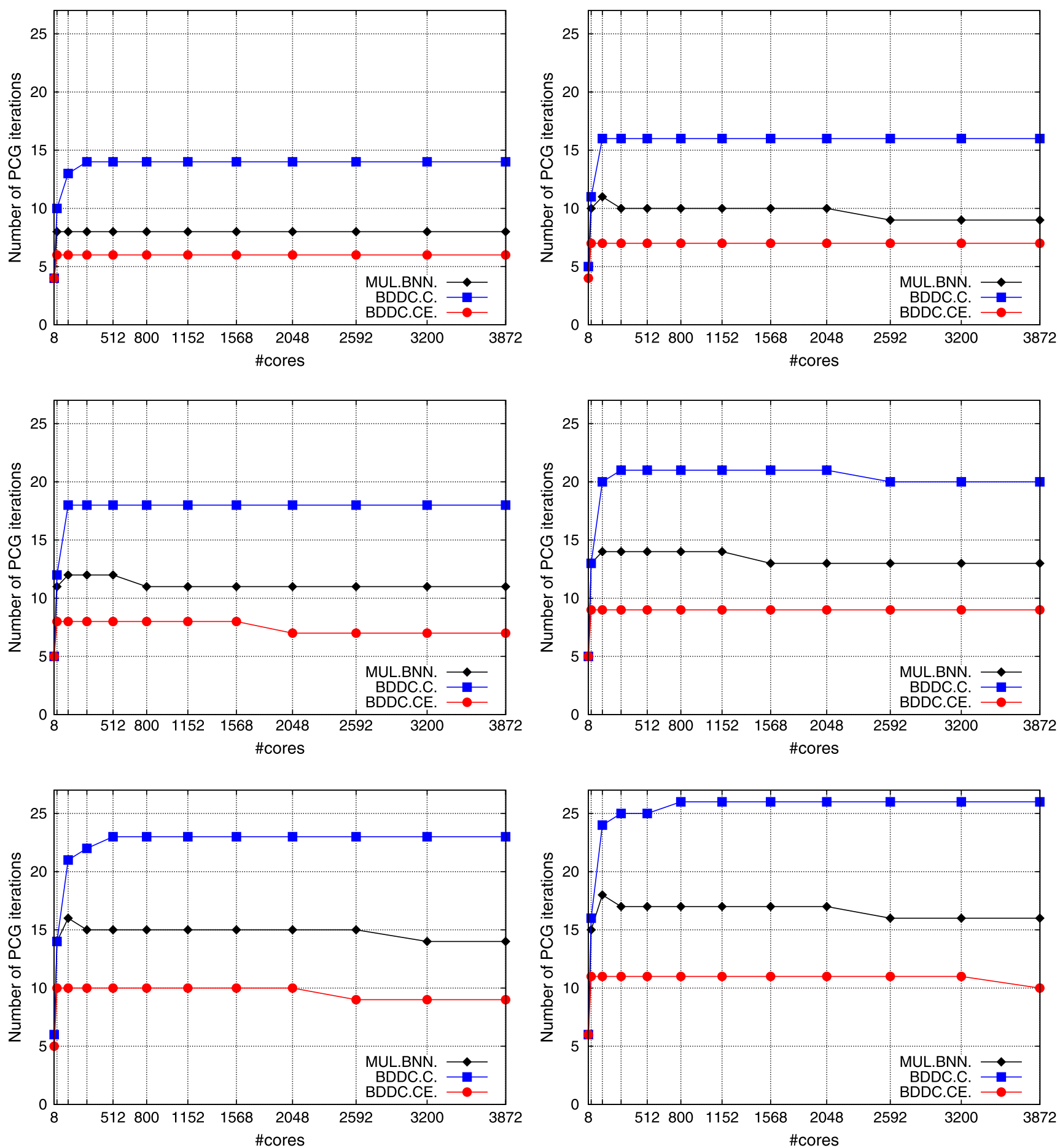

Fig. 7 Weak scalability for the number of PCG iterations of the multiplicative BNN and the BDDC(c) and BDDC(ce) solvers for the 2D Poisson problem on HPC-FF. From top to bottom and left to right $\frac{H}{h}=16,32,64,128,256,512$

dominates. The relative ranking of the methods is therefore determined by the extra cost required for the solution of the coarse-grid problem of each method, which has already been examined and properly justified in Sect. 4.3.1. However, a very nice observation is that for gradually larger $\frac{H}{h}$, the scalable part becomes more and more dominant, till the non-scalable component is completely masked, i.e., with $\frac{H}{h}=256,512$. Within this range, the winner method is the one with the least asymptotic number of PCG iterations, i.e., the BDDC(ce) solver, as illustrated in Fig. 7; a significant maximum improvement of $43 \%$ and $30 \%$ which comes from the use of (symmetric-PD) solvers can also be 


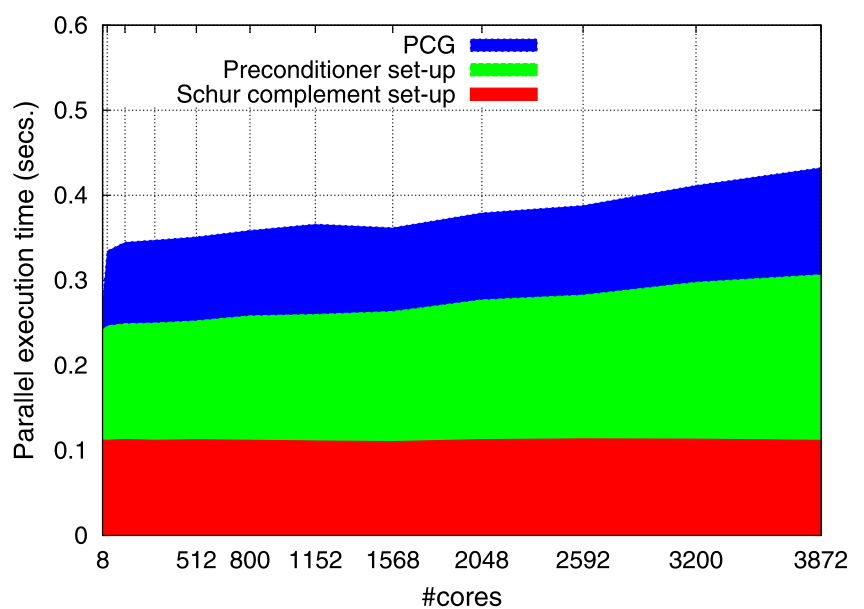

(a)

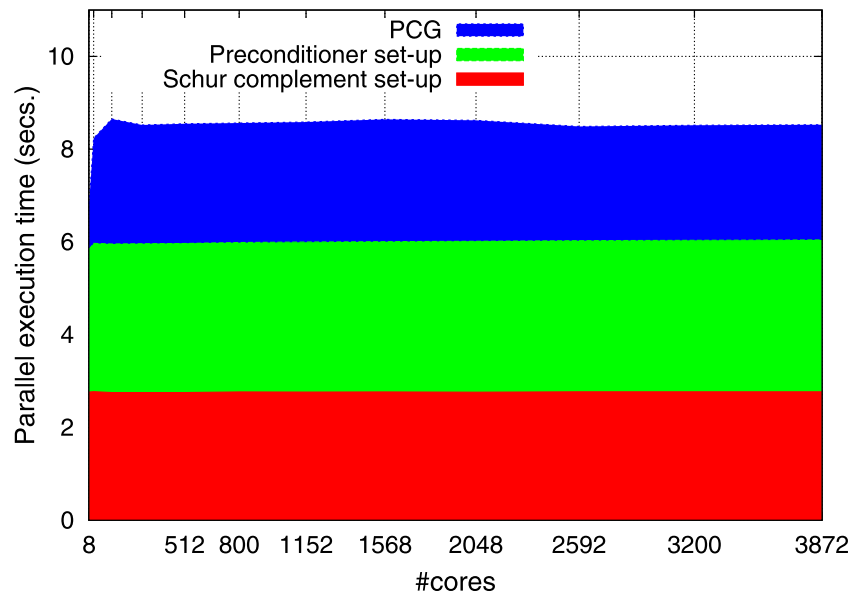

(c)

Fig. 8 Distribution of the total computation time among Schur complement and preconditioner set-up, and the iterative solution of the interface problem for 2D Poisson on HPC-FF. Multiplicative BNN and

observed within this range for the $\mathrm{BDDC}(\mathrm{c})$ and $\mathrm{BDDC}(\mathrm{ce})$ solvers, respectively. The number of cores can certainly be increased arbitrarily so that the coarse-grid component becomes dominant. However, a very remarkable conclusion from this study is that given the memory available per core on current distributed-memory machines, and the experimental evidence we have gathered so far, this is only expected to happen for simulations with several tens of thousands of cores. Besides, the margin of improvement for the coarse-grid preconditioning level is huge. In Fig. 7 it can also be observed no degradation of PCG convergence rates with $P$ and fixed $\frac{H}{h}$, and only a mild (i.e., logarithmic) grow with $\frac{H}{h}$ and $P$ fixed. This is a well-known property of BNN/BDDC solvers easily derived from the condition number bounds of the preconditioned operator (see Sects. 2.3 and 2.4).

Although not explicitly provided in Figs. 6 and 7, the NN-PCG solver required, for $\frac{H}{h}=16$, and $P=228$ and

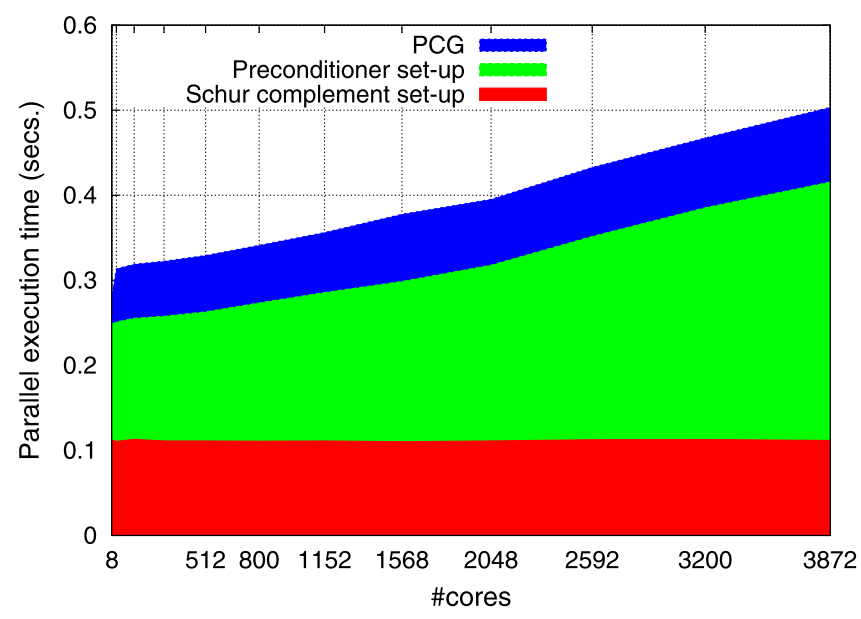

(b)

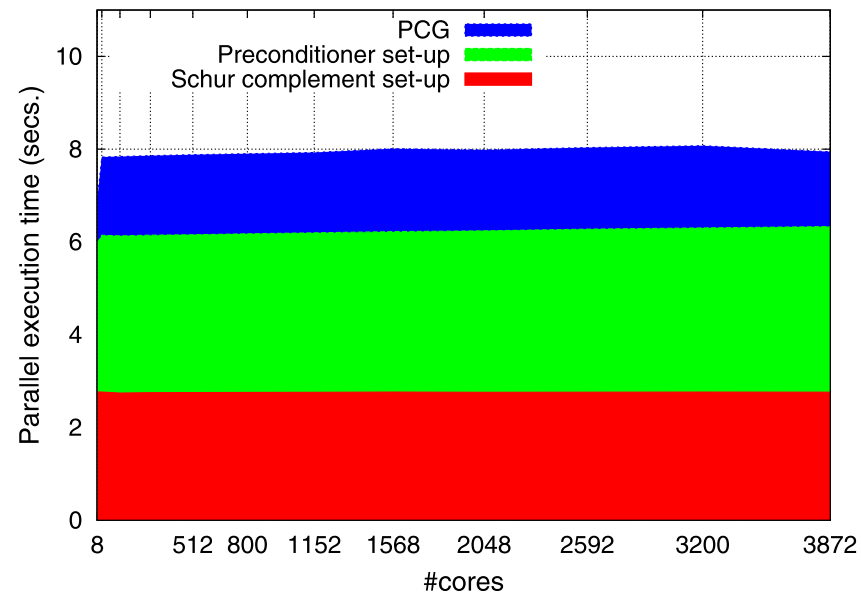

(d)

BDDC(ce) with $\frac{H}{h}=128\left((\mathbf{a})\right.$ and (b), respectively) and $\frac{H}{h}=512$ ((c) and (d), respectively)

$P=3872,0.18$ and 1.73 seconds, with 156 and 2207 PCG iterations, respectively. These numbers are significantly worse than those of the BNN/BDDC solvers. Therefore, even in the most favourable scenario for the NN-PCG solver in the experiment (i.e., the smallest local problem size considered), the extra cost associated with the coarse-grid correction more than pays off in terms of total computational time (due to the significant cut down in the number of iterations). For larger values of $\frac{H}{h}=16$, the gap among NN-PCG and the BDDC/BNN solvers becomes progressively larger, as predicted by Table 2 (as the term that depends on $\sqrt{P}$ is multiplied by a function that grows with $\frac{H}{h}$ ).

Figure 8 offers a complementary view of the weak scaling curves in Fig. 6. For $\frac{H}{h}=128$ (see Figs. 6(a) and (b)), the computation time of the preconditioner set-up and iterative phases grow with $P$ (the latter at a very moderate pace compared to the former as predicted by Sect. 4.2 and experimentally examined in Sect. 4.3.1). However, for $\frac{H}{h}=512$ (see 

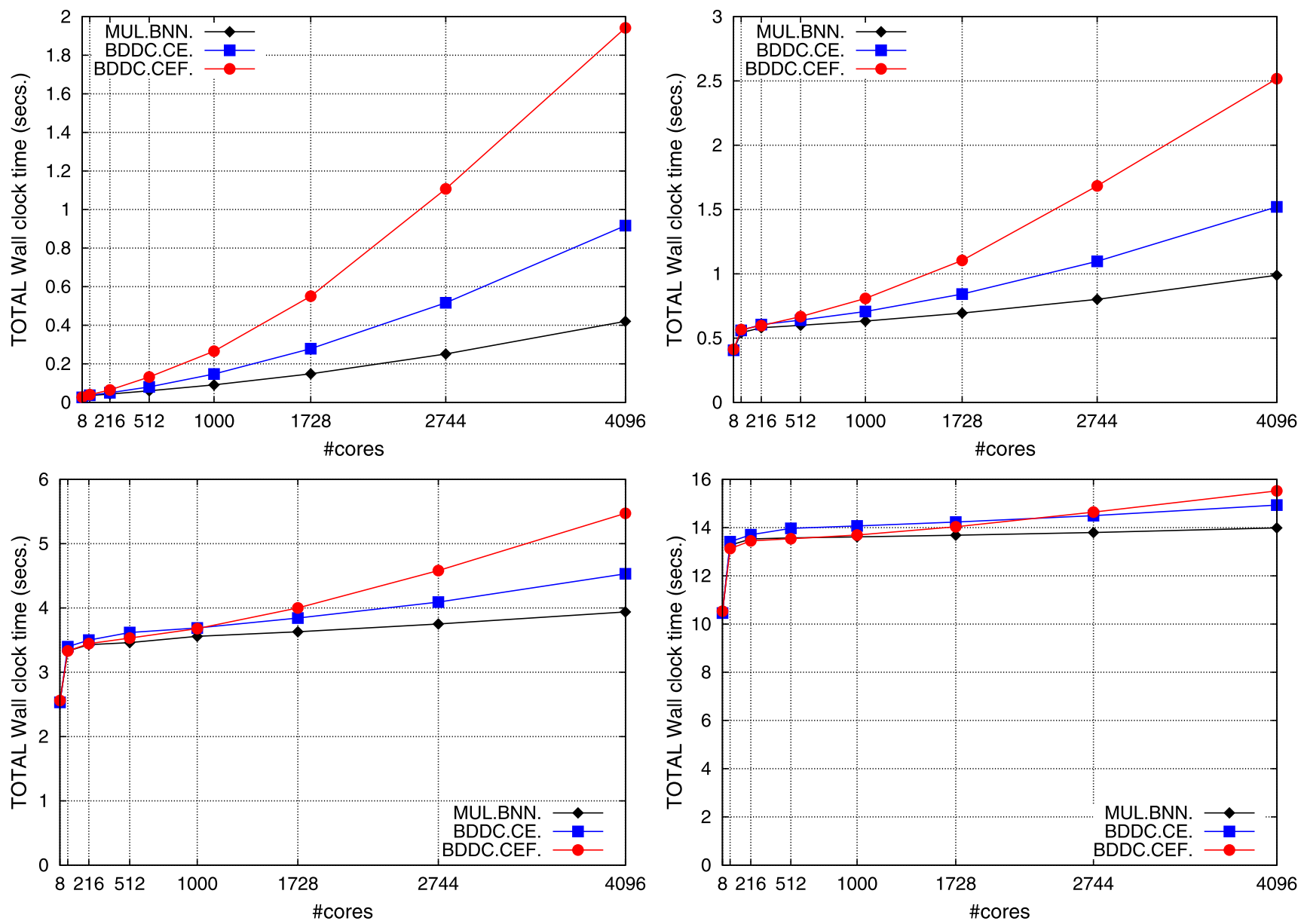

Fig. 9 Weak scalability for the total computation time of the multiplicative BNN (winner implementation) and the BDDC(ce) and BDDC(cef) solvers (winner implementation) for the 3D Poisson problem on HPC-FF. From top to bottom and left to right $\frac{H}{h}=10,20,30,40$

Figs. 6(c) and (d)), the computation time of the three phases is constant. An interesting observation for this "large" local problem size is that the computation time for preconditioner set-up is equivalent for the BNN and BDDC(ce) solvers. In the latter method, an extra number, proportional to the number of edge constraints, sparse forward/backward substitutions with the factor of $A_{R R}^{i}$ are required to build the Schur complement associated to edge constraints (see Sect. 3.2.2). However, this is only a constant and modest multiple, completely masked by the higher order of complexity of the sparse Cholesky factorization of $A_{R R}^{i}$. We stress, however, that this is no longer true if approximate solvers (e.g., AMG) are used as local solvers (due to their linear order of complexity).

\subsubsection{D Experiments}

Figures 9 and 10 compare the weak scalability for the total computation time and number of PCG iterations of the winner implementation of the multiplicative BNN solver and those of the winner implementation of the BDDC(ce) and
BDDC(cef) solvers. Figure 9 again reveals the two components of the weak scaling curves. For sufficiently "small" $\frac{H}{h}$ (e.g., $\frac{H}{h}=10,20$ ), the total computational time is dominated by computation and communication overheads related to the solution of the coarse-grid system. To be more precise, it is dominated by the sparse Cholesky factorization of the coarse-grid coefficient matrix: the shape of the curves in Fig. 9 resembles (particularly with large $P$ ) that of the curves in Fig. 5(b). For a very precise explanation of the relative ranking of the BNN/BDDC solvers for "small" $\frac{H}{h}$, we refer the reader to Sect. 4.3.2. For large $\frac{H}{h}$, the finegrid preconditioning component of the solvers dominates and the efficiency of the methods is very high due to their ability to keep the condition number bounded by a constant with $P$ and $\frac{H}{h}$ fixed (see Fig. 10). It is remarkable the nice scalability of the BNN compared to that of the BDDC solver in terms of computational time (see Fig. 9). For this latter method, a (mild) degradation of the weak scalability can already be observed for large $P$ even for the largest $\frac{H}{h}$. 

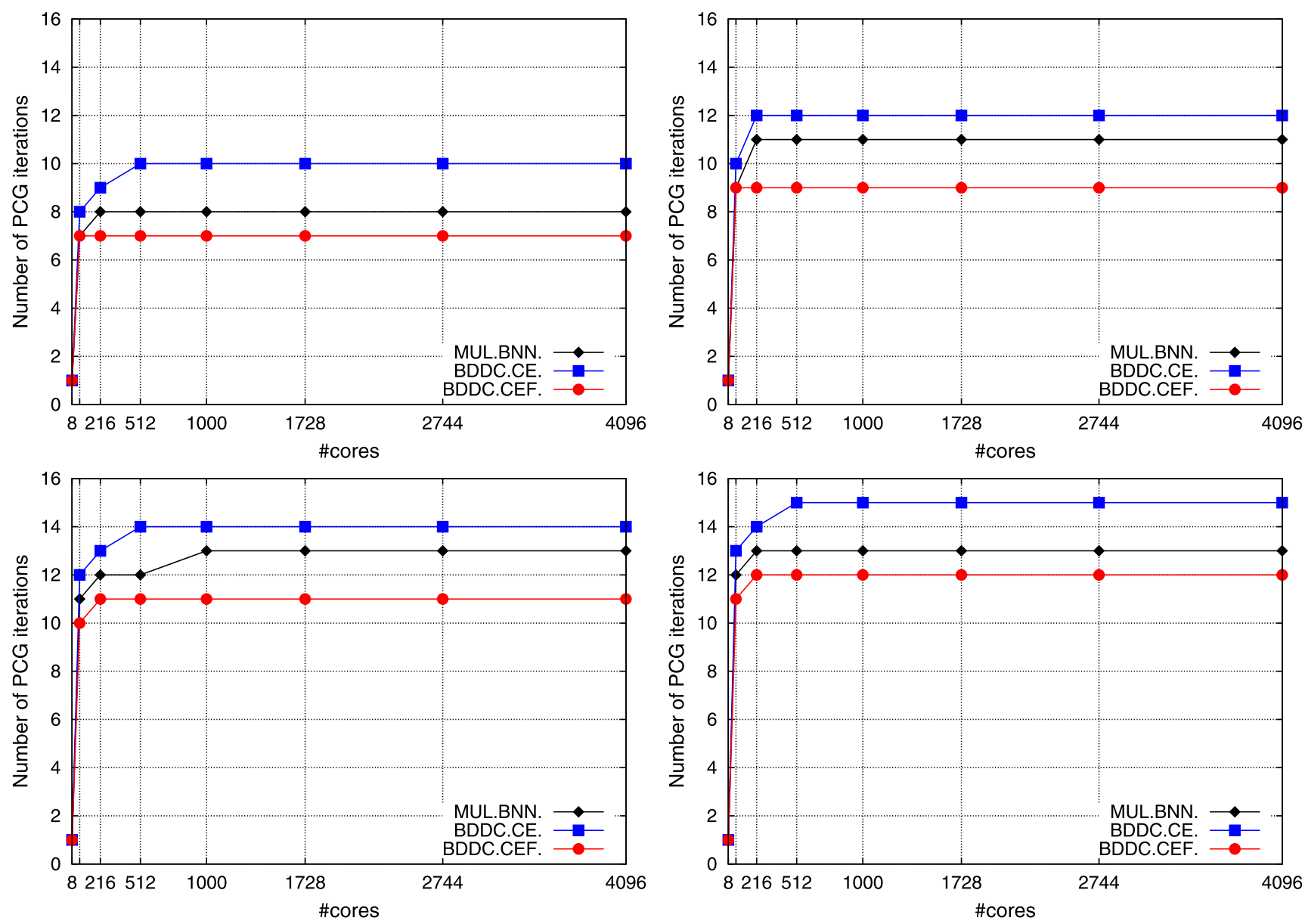

Fig. 10 Weak scalability for the number of PCG iterations of the multiplicative BNN and the BDDC(ce) and BDDC(cef) solvers for the 3D Poisson problem on HPC-FF. From top to bottom and left to right $\frac{H}{h}=10,20,30,40$

\section{Conclusions and Future Work}

In this work we have covered in detail the high-performance distributed-memory implementation of DD methods of balancing type. This comprehensive coverage presents a pool of hints and considerations that can be very useful for scientists that are willing to tackle large-scale distributed-memory machines using these methods. On the other hand, the paper presents a complete scalability study of BDDC/BNN preconditioners on a large-scale machine with up to 4096 cores. As far as we know, the state-of-the-art does not include any work that performs such study with these particular methods, at least with the degree of detail and up to the scale that are reached in this work. This scalability study answers the very interesting question of how far can the proposed MPI implementation go in the number of cores and the scale of the problem to still be within reasonable ranges of efficiency. The answer is up to dozens of thousands of computational cores in the solution of problems discretized with hundreds of millions FEs. Besides, the study has also precisely identified, quantified and justified which are the main sources of inefficiency and bottlenecks in our current implementation, namely communication and computation associated to the solution of the coarse-grid problem. In light of these conclusions, we have identified improvements that deserve further research in order to boost the current scalability of our MPI implementation, e.g., the use of approximate solvers (as AMG-preconditioned CG), multilevel BDD formulations (see [30]) or distributed memory coarse-grid solvers.

Acknowledgements This work has been funded by the European Research Council under the FP7 Programme Ideas through the Starting Grant No. 258443 - COMFUS: Computational Methods for Fusion Technology and the FUSSIM project (Ref. ENE2011-285556), financed by the Spanish Ministry of Science and Innovation (MICINN), under the programme "Proyectos de Investigación fundamental no orientada". A.F. Martín was also partially funded by the UPC postdoctoral grants under the programme "BKC-5-Atracció i Fidelització de talent al BKC". The authors thankfully acknowledge the computer resources, technical expertise and assistance provided by the Red Española de Supercomputación and the Juelich Supercomputing Centre in the exploitation of the HPC for Fusion (HPC-FF) under the EFDA HPC Implementing Agreement (EFDA (08) 39/4.1). 


\section{References}

1. Badia S, Codina R (2008) Algebraic pressure segregation methods for the incompressible Navier-Stokes equations. Arch Comput Methods Eng 15:343-369

2. Badia S, Martín AF, Príncipe J (2012) Enhanced balancing Neumann-Neumann preconditioning in computational fluid and solid mechanics. Int J Numer Methods Eng (in press)

3. Baker AH, Gamblin T, Schulz M, Yang UM (2011) Challenges of scaling algebraic multigrid across modern multicore architectures In: Parallel distributed processing symposium (IPDPS). 2011 IEEE international, pp 275-286

4. Balay S, Brown J, Buschelman K, Eijkhout V, Gropp WD, Kaushik D, Knepley MG, McInnes LC, Smith BF, Zhang H (2012) PETSc users manual. Technical report ANL-95/11, Argonne National Laboratory

5. Balay S, Brown J, Buschelman K, Gropp WD, Kaushik D, Knepley MG, McInnes LC, Smith BF, Zhang H (2012). PETSc web page. http://www.mcs.anl.gov/petsc

6. Brenner SC, Scott R (2010) The mathematical theory of finite element methods, 3rd edn. Springer, Berlin

7. Conceição D, Goldfeld P, Sarkis M (2007) Robust two-level lower-order preconditioners for a higher-order stokes discretization with highly discontinuous viscosities. In: High performance computing for computational science-VECPAR 2006, pp 319333

8. Davis TA (2006) Direct methods for sparse linear systems, vol 2. SIAM, Philadelphia

9. De Roeck YH, Le Tallec P (1991) Analysis and test of a local domain decomposition preconditioner. In: Fourth international symposium on domain decomposition methods for partial differential equations, $112 \mathrm{pp}$

10. Dohrmann CR (2003) A preconditioner for substructuring based on constrained energy minimization. SIAM J Sci Comput 25(1):246-258

11. Farhat C, Roux F-X (1991) A method of finite element tearing and interconnecting and its parallel solution algorithm. Int J Numer Methods Eng 32(6):1205-1227

12. Farhat C, Pierson K, Lesoinne M (2000) The second generation FETI methods and their application to the parallel solution of large-scale linear and geometrically non-linear structural analysis problems. Comput Methods Appl Mech Eng 184(2-4):333-374

13. Ferencz RM, Hughes TJR (1998) Iterative finite element solutions in nonlinear solid mechanics. In: Numerical methods for solids (part 3). Handbook of numerical analysis, vol VI

14. Filippone S, Buttari A (2012) Object-oriented techniques for sparse matrix computations in Fortran 2003. ACM Trans Math Softw 38(4):23:1-23:20

15. Filippone S, Colajanni M (2000) PSBLAS: a library for parallel linear algebra computation on sparse matrices. ACM Trans Math Softw 26(4):527-550

16. George A (1973) Nested dissection of a regular finite element mesh. SIAM J Numer Anal 10(2):345-363

17. Giraud L, Haidar A, Watson LT (2008) Parallel scalability study of hybrid preconditioners in three dimensions. Parallel Comput 34(6-8):363-379

18. Glowinski R, Wheeler MF (1988) Domain decomposition and mixed finite element methods for elliptic problems. In: First international symposium on domain decomposition methods for partial differential equations, pp 144-172

19. Goldfeld P (2003) Balancing Neumann-Neumann preconditioners for the mixed formulation of almost-incompressible linear elasticity. $\mathrm{PhD}$ thesis

20. Goldfeld P, Pavarino LF, Widlund OB (2003) Balancing Neumann-Neumann preconditioners for mixed approximations of heterogeneous problems in linear elasticity. Numer Math 95:283324
21. Gropp W, Lusk E, Skjellum A (1999) Using MPI: portable parallel programming with the message passing interface, vol 1. MIT Press, Cambridge

22. Gropp W, Lusk E, Thakur R (1999) Using MPI-2: advanced features of the message passing interface. MIT Press, Cambridge

23. Heroux MA, Willenbring JM (2003) Trilinos users guide. Technical report SAND2003-2952, Sandia National Laboratories

24. Heroux MA, Bartlett RA, Howle VE, Hoekstra RJ, Hu JJ, Kolda TG, Lehoucq RB, Long KR, Pawlowski RP, Phipps ET, Salinger AG, Thornquist HK, Tuminaro RS, Willenbring JM, Williams A, Stanley KS (2005) An overview of the Trilinos project. ACM Trans Math Softw 31(3):397-423

25. Hoefler T, Traff JL (2009) Sparse collective operations for MPI. In: Proceedings of the 2009 IEEE international symposium on parallel \& distributed processing, pp 1-8

26. Karypis G, Kumar V (1998) A fast and high quality multilevel scheme for partitioning irregular graphs. SIAM J Sci Comput 20(1):359-392

27. Lin PT, Shadid JN, Sala M, Tuminaro RS, Hennigan GL, Hoekstra RJ (2009) Performance of a parallel algebraic multilevel preconditioner for stabilized finite element semiconductor device modeling. J Comput Phys 228(17):6250-6267

28. Mandel J (1993) Balancing domain decomposition. Commun $\mathrm{Nu}-$ mer Methods Eng 9(3):233-241

29. Mandel J, Dohrmann CR (2003) Convergence of a balancing domain decomposition by constraints and energy minimization. Numer Linear Algebra Appl 10(7):639-659

30. Mandel J, Sousedík B, Dohrmann C (2008) Multispace and multilevel BDDC. Computing 83:55-85

31. Saad Y (1995) Data structures and algorithms for domain decomposition and distributed sparse matrix computations. Technical report 95-014, Department of Computer Science, University of Minnesota, Minneapolis, MN

32. Saad Y (2003) Iterative methods for sparse linear systems, 2nd edn. SIAM, Philadelphia

33. Saad Y, Sosonkina M (1999) Distributed Schur complement techniques for general sparse linear systems. SIAM J Sci Comput 21(4):1337-1356

34. Sahni O, Carothers CD, Shephard MS, Jansen KE (2009) Strong scaling analysis of a parallel, unstructured, implicit solver and the influence of the operating system interference. Sci Program 17(3):261-274

35. Sala M, Tuminaro R (2008) A new Petrov-Galerkin smoothed aggregation preconditioner for nonsymmetric linear systems. SIAM J Sci Comput 31(1):143-166

36. Schenk O, Gärtner K (2004) Solving unsymmetric sparse systems of linear equations with PARDISO. Future Gener Comput Syst 20(3):475-487

37. Schenk O, Gärtner K (2006) On fast factorization pivoting methods for sparse symmetric indefinite systems. Electron Trans Numer Anal 23:158-179

38. Sístek J, Sousedík B, Burda P, Mandel J, Novotný J (2011) Application of the parallel BDDC preconditioner to the Stokes flow. Comput Fluids 46(1):429-435

39. Strang G (2006) Linear algebra and its applications. Thomson Brooks/Cole, Belmont

40. Stüben K (2001) A review of algebraic multigrid. J Comput Appl Math 128(1-2):281-309

41. Tang J, Nabben R, Vuik C, Erlangga Y (2009) Comparison of twolevel preconditioners derived from deflation, domain decomposition and multigrid methods. J Sci Comput 39(3):340-370

42. Toselli A, Widlund O (2005) Domain decomposition methodsalgorithms and theory. Springer, Berlin

43. Vaněk P, Mandel J, Brezina M (1996) Algebraic multigrid by smoothed aggregation for second and fourth order elliptic problems. Computing 56:179-196 